\title{
Plethora of relativistic charged spheres: The full spectrum of Guilfoyle's static, electrically charged spherical solutions
}

\author{
José P. S. Lemos \\ Centro Multidisciplinar de Astrofísica - CENTRA, \\ Departamento de Física, \\ Instituto Superior Técnico - IST, \\ Universidade de Lisboa - UL, \\ Avenida Rovisco Pais 1, 1049-001 Lisboa, Portugal \\ Email: joselemos@ist.utl.pt \\ Vilson T. Zanchin \\ Centro de Ciências Naturais e Humanas, \\ Universidade Federal do $A B C$, \\ Avenida dos Estados, 5001, \\ 09210-580 Santo André, São Paulo, Brazil \\ Email: zanchin@ufabc.edu.br
}

\begin{abstract}
We show that Guilfoyle's exact solutions of the Einstein-Maxwell equations for spherical symmetric static electrically charged matter with a Reissner-Nordström exterior possess a bewildering plethora of different types of solutions. For the parameter space of the solutions we use two normalized variables, $q^{2} / R^{2}$ and $r_{0} / R$, where $q$ is the total electric charge, $r_{0}$ is the radius of the object, and $R$ is a length representing the square root of the inverse energy density of the matter. The two other parameters, the mass $m$ and the Guilfoyle parameter $a$, both dependent on $q, r_{0}$ and $R$, are analyzed in detail. The full parameter space of solutions $q^{2} / R^{2} \times r_{0} / R$ is explored with the corresponding types of solutions being identified and analyzed. The different types of solutions are regular charged stars, including charged dust stars and stars saturating the Buchdahl-Andréasson bound, quasiblack holes, regular charged black holes with a de Sitter core, regular black holes with a core of phantom charged matter, other exotic regular black holes, Schwarzschild stars, Schwarzschild black holes, Kasner spacetimes, pointlike and planar naked singularities, and the Minkowski spacetime. Allowing for $q^{2}<0$, in which case it is not possible to interpret $q$ as electric charge, also yields new solutions, some of which are interesting and regular, others are singular. Some of these types of solutions as well as the matter properties have been previously found and studied, here the full spectrum being presented in a unified manner.
\end{abstract}

PACS numbers: 04.70.Bw, 04.20.Jb, 04.40.Nr

Keywords: Relativistic charged spheres, regular black holes; quasiblack holes

\section{INTRODUCTION}

Guilfoyle found an amazing class of electric spherically symmetric interior fluid solutions joined to a ReissnerNordström outer spacetime [1]. The considerable potential of these starlike solutions has been understood by Lemos and Zanchin, who studied the specific aspects of its fluid properties [2], analyzed quasiblack holes as the frozen stars [3], tested the sequence of configurations that saturate the Buchdahl-Andréasson bound for gravitational collapse [4], and found regular black holes within the whole set of solutions [5]. From these previous works it is clear that Guilfoyle's solutions possess a richness of solutions that must be explored in full. We thus embark on a full study of the properties of the bewildering variety of this class of Guilfoyle's star solutions, i.e., we give the full spectrum of the solutions.

The motivation for the Guilfoyle's solutions [1] starts with the work of Weyl [6] who has considered static Einstein-Maxwell systems. Static Einstein-Maxwell sys- tems have played an important role in understanding the structure of extremely compacted objects in general relativity. The relative simplicity of the resulting system of differential equations allied to the rich analytical properties resulting from the coupling of the electrostatic Maxwell and the gravitational fields are a reason for such a relevance. In Weyl [6] a functional relation between the metric component $g_{t t} \equiv B$ and the electric potential $\phi$, $B=B(\phi)$, the Weyl ansatz, is assumed to exist. In electrovacuum he found that if there is a relation, it must be of a quadratic form $B(\phi)=(-\epsilon \phi+b)^{2}+c$, the Weyl relation, where $\epsilon= \pm 1, b$ and $c$ are arbitrary constants, and we use units such that the speed of light and the Newton's gravitational constant equal unity. Majumdar [7] showed that such a quadratic relation exists for generic static spacetimes, not only for the axisymetric ones that Weyl had used [6]. When $c=0$ the relation between the potentials is a perfect square and in that case Majumdar 7] and Papapetrou [8] showed that the solution is the vacuum solution exterior to some static charged dust distribution in which the gravitational attraction balances 
exactly the electric repulsion. When the MajumdarPapapetrou relation holds, i.e., $B(\phi)=(-\epsilon \phi+b)^{2}$, and besides electric fields there is matter one can further show [7, 8] that the pressure of the matter content should vanish and that the charge density $\rho_{\mathrm{e}}$ and the energy density $\rho_{\mathrm{m}}$ of the charged dust must obey $\rho_{\mathrm{e}}=\epsilon \rho_{\mathrm{m}}$, i.e., the matter is extremal matter (see also [9]). One can then construct interior Majumdar-Papapetrou matter solutions matched to an exterior Majumdar-Papapetrou vacuum solution. For spherical symmetry these solutions are the Bonnor stars [10, 11], with an exterior extremal Reissner-Nordström spacetime. On the other hand, if one wants to include fluid pressure into the solutions, the Majumdar-Papapetrou relation cannot hold and the Weyl relation runs into difficulties. So a new route, other than Weyl's and Majumdar and Papapetrou's, was taken. The idea was to generalize the interior spherical symmetric Schwarzschild solution, a very interesting and useful solution, and this was done by Cooperstock and de la Cruz [12] and Florides [13]. They found electric star solutions. Guilfoyle final motivation was to use $B=B(\phi)$, the Weyl ansatz, and see which functional forms would work that would give solutions with pressure [1]. A simple one is $B(\phi)=a(-\epsilon \phi+b)^{2}$, with $a$ a new parameter, the Guilfoyle parameter, in which case the Cooperstock and de la Cruz [12] star is reproduced as a particular solution, but there are others also used in [1].

The motivation for the work [2] is related to the study of the fluid properties that obey a Weyl ansatz $B=B(\phi)$, namely, fluids that obey either a Weyl relation, a Majumdar-Papapetrou relation, or a Guilfoyle relation. The work [2] is based, besides [1], in the works of Das [14], De and Raychaudhuri [15], Gautreau and Hoffman [16], and Bonnor [17]. Das [14], showed that if the ratio $\rho_{\mathrm{e}} / \rho_{\mathrm{m}}=\epsilon$ is assumed, then the relation between potentials must be of the form of the Majumdar-Papapetrou relation $B=(-\epsilon \phi+b)^{2}$. De and Raychaudhuri [15] went a step further and generalized this theorem assuming more general conditions, such as there is a closed equipotential within the charged dust fluid with no singularities, holes, or another kind of matter, then the charged dust fluid corresponds to a Majumdar-Papapetrou solution. The inclusion of matter with pressure into the Weyl relation was first considered by Gautreau and Hoffman [16]. They verified that if the metric potential $B$ is given by the Weyl relation $B(\phi)=(-\epsilon \phi+b)^{2}+c$, then the perfect fluid satisfies the condition $\rho_{\mathrm{e}}(\epsilon \phi-b)=-\epsilon\left(\rho_{\mathrm{m}}+3 p\right) \sqrt{B}$. Bonnor 17] also displayed some theorems for these type of systems. In [2] the Gautreau and Hoffman [16] result was generalized to systems obeying a Guilfoyle relation, namely, $a \rho_{\mathrm{e}}(\epsilon \phi-b)=-\epsilon\left(\rho_{\mathrm{m}}+3 p+\epsilon(1-a) \rho_{\mathrm{em}}\right) \sqrt{B}$, where $\rho_{\mathrm{em}}$ is the electromagnetic energy density, and some new results drawn upon [1] were extended.

The motivation for [3] was the search for quasiblack holes within Guilfoyle's stars [1]. Quasiblack holes are stars on the verge of becoming black holes, stars whose boundary to the exterior is the event horizon. Quasiblack holes have been reported in [18 -20]. Their properties were studied in 21 25]. Previous results with electric stars with quasiblack hole properties were reported by Bonnor [26] (see also 10]) and in 27 29], in [28] a stability analysis of the solutions was performed. Quasiblack holes with non-Abelian gauge fields were found in [30] and quasiblack holes with rotation have also been found in [31]. Now, in [1] some simple cases of solutions were displayed, notably solutions which obeyed the condition $m>\frac{q^{2}}{r_{0}}$, where $m, q$, and $r_{0}$ are the mass, electric charge and radius of the star, respectively. By extending these solutions to the limiting case $m=\frac{q^{2}}{r_{0}}$ quasiblack holes with pressure, i.e., relativistic charged spheres as frozen stars, were found within Guilfoyle's solutions [3].

The motivation for [4] was to search whether Guilfoyle's stars [1] saturate the Buchdahl-Andréasson bound. Buchdahl [32] showed that, under some physical assumptions, a star made of a perfect fluid would obey a compactness bound $r_{0} / m \geq 9 / 4$. The interior Schwarzschild solution obeys this bound, the equality appearing when the star's central pressure goes to infinity. For electrically charged matter it was found by Andréasson [33] that there was also a bound $r_{0} / m \geq$ $9 /\left(1+\sqrt{1+3 q^{2} / r_{0}^{2}}\right)^{2}$. While Buchdahl's proof is appropriate for stars, Andréasson's proof is appropriate for thin shells, and he demonstrated that indeed thin shells saturate his bound, the Buchdahl-Andréasson bound. In [4] it was shown that Guilfoyle's stars also saturate the Buchdahl-Andréasson bound. There are other electric stars that do not saturate the bound, although they almost do [34, 35].

The motivation for [5] was to search for regular black holes within Guilfoyle's solutions [1]. Regular black holes were envisioned by Bardeen [36] and subsequent works displayed other type of solutions and their properties $37-$ 42]. In [5] it was shown that indeed there are also regular black holes in Guilfoyle's solutions [1].

Here we perform a full analysis and make a classification of the plethora of Guilfoyle's solutions for the relation $B(\phi)=a(-\epsilon \phi+b)^{2}$, this set was named Ia in [1]. We explore the whole parameter space and try to interpret all the corresponding solutions. In passing we will deal with imaginary electric charges, a concept that appeared first in the context of particle solutions in general relativity and is linked to the Einstein-Rosen bridge [43], see also [44], and we will also deal with Kasner metrics that arise in the interior of black holes [45, 46].

The paper is organized as follows. In Sec. III the exact Guilfoyle's set of solutions with the ansatz $B(\phi)=$ $a(-\epsilon \phi+b)^{2}$ is displayed in full, with its interior, exterior, and junction surface. We also display the constraints the parameters should satisfy. In Sec. III a general analysis 
of the mass $m$ and the Guilfoyle parameter $a$ of the solutions in terms of the other free parameters is done. In Sec. IV we show that it is useful to visualize the parameter space regions in a plot $r_{0} / R \times q^{2} / R^{2}$. The regions, i.e., areas in this parameter space, then appear naturally according to the characteristics of the respective solutions and we classify them and name the corresponding objects, such as normal stars, tension stars, regular black holes, singular black holes and others. A brief description of the kinds of solutions contained in each region is also given. In Sec. $\mathrm{V}$ we study the solutions belonging to the boundaries of the regions, i.e., areas, described in the previous section. These regions are lines and points in the parameter space on which interesting solutions can be found. Finally, in Sec. VI we conclude.

\section{GUILFOYLE'S SOLUTIONS AND CONSTRAINTS ON THE SOLUTIONS}

\section{A. Spherical static Weyl-Guilfoyle systems and equations}

The spacetime is static and spherically symmetric with the metric written in the form

$$
d s^{2}=-B(r) d t^{2}+A(r) d r^{2}+r^{2}\left(d \theta^{2}+\sin ^{2} \theta d \varphi^{2}\right),
$$

where $(t, r, \theta, \varphi)$ are spherical symmetric spacetime coordinates, and the functions $A$ and $B$ depend only on the radial coordinate $r$.

The source is a static charged fluid distribution with spherical symmetry. The fluid has an energy density $\rho_{\mathrm{m}}(r)$, an isotropic pressure $p(r)$, an electric charge density $\rho_{\mathrm{e}}(r)$, and a time component of the four-velocity given by

$$
U_{t}=-\sqrt{B(r)}
$$

the other components of the four-velocity being zero. The electromagnetic gauge potential has only one nonzero component, given by

$$
\mathcal{A}_{t}=-\phi(r)
$$

with $\phi(r)$ being the electric potential.

The mass $M(r)$ inside a sphere of radius $r$ is defined as

$$
M(r)=\int_{0}^{r} 4 \pi r^{2}\left(\rho_{\mathrm{m}}(r)+\frac{Q^{2}(r)}{8 \pi r^{4}}\right) d r+\frac{Q^{2}(r)}{2 r},
$$

and the electric charge $Q(r)$ inside a sphere of radius $r$ is defined as

$$
Q(r)=4 \pi \int_{0}^{r} \rho_{\mathrm{e}}(r) \sqrt{A(r)} r^{2} d r
$$

These two quantities, $M(r)$ and $Q(r)$, are auxiliary and important quantities.

Now, we impose a Weyl ansatz into the system, i.e., the metric potential $B(r)$ and the electric potential $\phi(r)$ are functionally related through some relation $B=B(\phi)$, or in detail $B(r)=B(\phi(r))$. Here we want to study systems that obey the Guilfoyle relation [1] (see also [2]), namely, $B(r)=a[-\epsilon \phi(r)+b]^{2}$, where $\epsilon= \pm 1$, and $a$ and $b$ being arbitrary constants. Without loss of generality one can set $b=0$, since it can be absorbed into the electric potential $\phi$. Thus, the Guilfoyle relation can be written as

$$
B(r)=a \phi^{2}(r),
$$

with $a$ being called the Guilfoyle parameter.

The Einstein-Maxwell equations provide the set of equations for this system. The gravitational part of the equations yields the relations,

$$
\begin{aligned}
& \frac{B^{\prime}(r)}{B(r)}+\frac{A^{\prime}(r)}{A(r)}=8 \pi r A(r)\left[\rho_{\mathrm{m}}(r)+p(r)\right], \\
& \left(\frac{r}{A(r)}\right)^{\prime}=1-8 \pi r^{2}\left(\rho_{\mathrm{m}}(r)+\frac{Q^{2}(r)}{8 \pi r^{4}}\right),
\end{aligned}
$$

with a prime denoting the derivative with respect to the radial coordinate $r$ (units in which the gravitational constant and the speed of light are set to one are used). The definition of the total charge $Q(r)$ inside $r$, see Eq. (5), gives that the only nontrivial component of the Maxwell equation is $Q(r)=r^{2} \phi^{\prime}(r) / \sqrt{B(r) A(r)}$, where an integration constant has been put to zero. The electric potential $\phi(r)$ can be written in terms of $B(r)$ as $\epsilon \phi(r)=\sqrt{B(r) / a}$, see Eq. (6). Then, Eq. (5), can be written as

$$
Q(r)=\frac{-\epsilon r^{2} B^{\prime}(r)}{2 \sqrt{a A(r)} B(r)} .
$$

Once one has the metric functions $B(r)$ and $A(r)$ one obtains the electric charge distribution through Eq. (9). Equations (7)-(9) provide the equations for Weyl-Guilfoyle systems.

\section{B. Guilfoyle's solutions}

\section{Interior solution}

We assume that the interior solution extends from $r=$ 0 up to $r=r_{0}$. Guilfoyle's solutions are found under the additional assumption that the effective energy density $\rho_{\mathrm{m}}(r)+Q^{2}(r) / 8 \pi r^{4}$ is a constant, i.e.,

$$
8 \pi \rho_{\mathrm{m}}(r)+\frac{Q^{2}(r)}{r^{4}}=\frac{3}{R^{2}}
$$


where $R$, is a length parameter associated to the inverse of the total energy density. Through the junction conditions of the metric at the surface $r_{0}$, one finds that $R$ is to be related to the parameters of the exterior solution, namely, the total mass $m$ and the total charge $q$. The equation of state (10) is a generalization of the interior Schwarzschild solution equation of state to include electrically charged matter and had been proposed previously [12, 13]. With this additional assumption provided by (10), one can find that Guilfoyle's solutions [1] are given by

$$
\begin{aligned}
& A(r)=\left(1-\frac{r^{2}}{R^{2}}\right)^{-1} \\
& B(r)=\left[\frac{(2-a)^{2}}{a^{2}} F^{2}(r)\right]^{a /(a-2)}, \\
& \phi(r)=\epsilon \sqrt{\frac{B(r)}{a}}, \\
& 8 \pi \rho_{\mathrm{m}}(r)=\frac{3}{R^{2}}-\frac{a}{(2-a)^{2}} \frac{k_{0}^{2}}{R^{4}} \frac{r^{2}}{F^{2}(r)}, \\
& 8 \pi p(r)=-\frac{1}{R^{2}}+\frac{a}{(2-a)^{2}} \frac{k_{0}^{2}}{R^{4}} \frac{r^{2}}{F^{2}(r)}+ \\
& +\frac{2 a}{2-a} \frac{k_{0}}{R^{2}} \frac{\sqrt{1-\frac{r^{2}}{R^{2}}}}{F(r)} \text {, } \\
& 4 \pi \rho_{\mathrm{e}}(r)=\frac{\epsilon \sqrt{a}}{2-a} \frac{k_{0}^{2}}{R^{4}} \frac{r^{2}}{F^{2}(r)}\left(1+\frac{3 F(r) \sqrt{1-\frac{r^{2}}{R^{2}}}}{k_{0} r^{2}}\right)
\end{aligned}
$$

The functions $M(r)$ and $Q(r)$ defined in Eqs. (4) and (5), respectively, are then

$$
\begin{gathered}
M(r)=\frac{r^{3}}{2 R^{2}}+\frac{a}{2(2-a)^{2}} \frac{k_{0}^{2}}{R^{4}} \frac{r^{5}}{F^{2}(r)}, \\
Q(r)=\frac{\epsilon \sqrt{a}}{2-a} \frac{k_{0}}{R^{2}} \frac{r^{3}}{F(r)} .
\end{gathered}
$$

The function $F(r)$ has the following definition

$$
F(r)=k_{0} \sqrt{1-\frac{r^{2}}{R^{2}}}-k_{1}
$$

with the integration constants $k_{0}$ and $k_{1}$ given by

$$
k_{0}=\frac{R^{2}}{r_{0}^{2}}\left(\frac{m}{r_{0}}-\frac{q^{2}}{r_{0}^{2}}\right)\left(1-\frac{r_{0}^{2}}{R^{2}}\right)^{-1 / a},
$$

$$
k_{1}=k_{0} \sqrt{1-\frac{r_{0}^{2}}{R^{2}}}\left[1-\frac{a}{2-a} \frac{r_{0}^{2}}{R^{2}}\left(\frac{m}{r_{0}}-\frac{q^{2}}{r_{0}^{2}}\right)^{-1}\right]
$$

where

$$
\begin{gathered}
m \equiv M\left(r_{0}\right), \\
q \equiv Q\left(r_{0}\right) .
\end{gathered}
$$

The constants $k_{0}$ and $k_{1}$ were found through the junction conditions. In this work we are interested in all possible values for the parameter $a,-\infty<a<\infty$. The limiting case $a \rightarrow \pm \infty$ yields the uncharged, $q=0$, Schwarzschild interior solution.

\section{Exterior solution}

For the external region, $r>r_{0}$, the solution of the Einstein-Maxwell field equations, Eqs. (7)-(9), is the Reissner-Nordström solution, i.e.,

$$
\begin{gathered}
A(r)=\frac{1}{1-\frac{2 m}{r}+\frac{q^{2}}{r^{2}},} \\
B(r)=\frac{1}{A(r)}=1-\frac{2 m}{r}+\frac{q^{2}}{r^{2}}, \\
\phi(r)=\frac{q}{r}, \\
\rho_{\mathrm{m}}(r)=0, \\
p(r)=0, \\
\rho_{\mathrm{e}}=0 .
\end{gathered}
$$

The functions $M(r)$ and $Q(r)$ are simply

$$
\begin{gathered}
M(r)=m, \\
Q(r)=q,
\end{gathered}
$$

again, with $m$ and $q$ being the total mass and total charge of the exterior spacetime. By continuity, one finds that at $r=r_{0}$, the metric functions are $B\left(r_{0}\right)=1 / A\left(r_{0}\right)=$ $1-2 m / r_{0}+q^{2} / r_{0}^{2}$. One must also have $\phi\left(r_{0}\right)=q / r_{0}$, $M\left(r_{0}\right)=m$, and $Q\left(r_{0}\right)=q$. Such a spherical electrovacuum spacetime has two radii associated with it, the gravitational and the Cauchy radii, given in terms of $m$ and $q$. The gravitational radius is given by

$$
r_{+}=m+\sqrt{m^{2}-q^{2}} .
$$

It is the horizon radius if there is a black hole. The Cauchy radius is given by

$$
r_{-}=m-\sqrt{m^{2}-q^{2}} .
$$




\section{Junction conditions}

The matching between the interior and the exterior solution is done at the boundary surface $r=r_{0}$. At this boundary the metric should be continuous. So, by making the junction of the interior metric function $g_{r r}=A(r)$ in Eq. (11) to the $g_{r r}$ coefficient of the exterior metric function given by Eq. (25), one finds [1] that

$$
m=\frac{r_{0}}{2}\left(\frac{r_{0}^{2}}{R^{2}}+\frac{q^{2}}{r_{0}^{2}}\right) .
$$

An additional junction condition has to be imposed at $r=r_{0}$, namely, one must impose the continuity of the $g_{t t}=B(r)$ metric coefficient and the continuity of its first radial derivative to obtain

$$
a=\frac{r_{0}^{2}}{4 q^{2}}\left(\frac{r_{0}^{2}}{R^{2}}-\frac{q^{2}}{r_{0}^{2}}\right)^{2}\left(1-\frac{r_{0}^{2}}{R^{2}}\right)^{-1}
$$

where the fact that $Q\left(r_{0}\right)=q$ was also taken into account. The constants $k_{0}$ and $k_{1}$ in Eqs. (20)-(21) have been also found through the junction conditions.

\section{Some constraints}

If we restrict the solutions to be static spheres, the condition $r_{0}<R$ has to be obeyed. This condition has been imposed in [1]. On the other hand, when the matching surface $r_{0}$ is pushed to $R$ one finds regular black holes whose central core of charged matter fills up to the Cauchy horizon $r_{0}=r_{-}=R$ [4]. Therefore, we impose the constraint

$$
r_{0} \leq R
$$

In 1] it was displayed solutions satisfying some conditions, $m \geq|q|$ and $r_{0}>r_{+}$, which implied $m>q^{2} / r_{0}$. Also some energy conditions were imposed. Here we release all these constraints, except Eq. (36), and look at the whole space of parameters. We give a physical interpretation to the resulting solutions. Clearly, there is a large region in the parameter space for which there are solutions, indeed there is a plethora of many interesting solutions.

\section{Further considerations}

Let us briefly mention some of the territory in the space of solutions we are going to explore.

First, there is the special case $a=1$, not considered by Guilfoyle [1]. For the ansatz $B(r)=a \phi^{2}(r)$, Eq. (6), with $a=1$ one has the case with zero pressure, i.e., it is the Majumdar-Papapetrou ansatz for electrically charged fluids [7, 8]. When this Majumdar-Papapetrou matter is joined into a spherically symmetric vacuum one gets the Bonnor stars with $q=m$ [9, 10].

Second, for $a \neq 1$ and $a>0$, one finds from Eq. (34) that there is the possibility in which the equality $m r_{0}=$ $q^{2}$ holds, which can yield, as a particular case, the relation $q=m$, an extremal solution. This case has also not been considered in [1]. This limit, $m r_{0}=q^{2}$ and $q=m$ is the quasiblack hole limit [3]. Since the inequality $a \neq 1$ holds in this case, it means the corresponding quasiblack hole is made of a fluid with nonzero pressure. As we will see, in fact, this point in the parameter space, namely, $q=r_{0}=m$, is degenerated.

Third, another region in the parameter space, also not explored in the original paper [1], corresponds to extremely compact objects [4]. These objects are found by allowing the central pressure to assume arbitrarily large values and the objects saturate the Buchdahl-Andréasson bound [32, 33].

The above mentioned solutions are valid for all $a>0$, with the $a \rightarrow \infty$ needing a special treatment. The case $a=0$ is also special and requires a careful analysis in order to check if there are solutions in such a limit. If one allows $a<0$ then other interesting solutions may be found. In the following we study all the possibilities.

\section{GENERAL ANALYSIS, ANALYSIS OF THE PARAMETERS $m$ AND $a$, AND DISPLAY OF SOME SPECIAL CASES}

\section{A. General analysis}

1. The range of the parameters of the two-dimensional parameter space $q^{2} / R^{2} \times r_{0} / R$

These solutions have five parameters, namely, $a, m$, $r_{0}, R$, and $q$. Taking into account the two relations (34) and (35), one can consider that the free parameters of the model are three, we choose them to be the electric charge $q$, the radius of the star $r_{0}$, and the energy density parameter $R$. In the respective parameter space, these parameters in principle have the following range: $0 \leq r_{0}<\infty,-\infty<q<\infty$, and $0 \leq R<\infty$. The choice for the range of $r_{0}$ is obvious. The choice for the range of $q$ is based in the usual physical interpretation of the electric charge. However, we will do differently, and will allow $q$ to assume imaginary values, i.e., we will allow $q^{2}<0$. The interpretation of the parameter $R$, following its definition from Eq. (10), is that large $R$ means low energy densities while small $R$ represents large energy 
densities. The limiting value $R \rightarrow 0$ may be interpreted as a singular pointlike solution, while $R \rightarrow \infty$ represents empty space. To display in a clear way the spectrum of solutions we can do better, and without loss of generality, display the solutions in a two-dimensional parameter space instead of in the three-dimensional space spanned by $r_{0}, q$, and $R$.

For this let us give arguments to choose the twodimensional dimensionless parameter space $q^{2} / R^{2} \times r_{0} / R$ as the most interesting choice and also give the range of $q^{2} / R^{2}$ and $r_{0} / R$.

We consider the dimensionless ratio $q^{2} / R^{2}$ as a first free parameter. Now, the constraint (36) together with Eqs. (34)-35) imply $m$ and $a$ may be zero or negative only if $q^{2}$ is allowed to be negative. So, we may consider the dimensionless ratio $q^{2} / R^{2}$ with a range of $q^{2} / R^{2}$ not restricted, i.e.,

$$
-\infty<\frac{q^{2}}{R^{2}}<\infty
$$

We are thus allowing for an imaginary electric charge. The range of $q^{2}$ is the same as the range of $q^{2} / R^{2}$. We use the usual convention for the modulus of the electric charge, i.e., for $q^{2}<0$ we denote $|q|=\sqrt{\left|q^{2}\right|}$ and for $q^{2}>0$ we denote $|q|=\sqrt{q^{2}}$.

We consider the dimensionless ratio $r_{0} / R$ as a second free parameter. The constraint (36) together with the size of the charged matter distributions, which is given by its radius $r_{0}$ and the total energy density, codified by the parameter $R$, are related to the compactness of the objects. For instance, large energy densities may be obtained by taking a fixed mass $m$ and diminishing $r_{0}$, or by fixing $r_{0}$ and diminishing $R$. Moreover, the metric functions depend only on the ratio $r_{0} / R$, and, of course, on $r / R$. Hence, it is interesting to consider the ratio $r_{0} / R$ as the true free parameter of the model. This, together with the constraint $r_{0} \leq R$ imposed by the model, Eq. (36), implies that the range of the $r_{0} / R$ parameter is

$$
0 \leq \frac{r_{0}}{R} \leq 1
$$

The parameter space $q^{2} / R^{2} \times r_{0} / R$ is therefore twodimensional. The range of the parameters given in Eqs. (37) and (38) defines then a strip in the twodimensional parameter space. A preliminary analysis will be performed on the parameters $m$ and $a$ in terms of the free parameters $q^{2} / R^{2}$ and $r_{0} / R$. Figures 1 to 4 will help in the understanding of the whole spectrum of solutions.

For the study that follows, it shall be convenient to divide the parameter space into two sectors: (I) $q^{2} / R^{2}>$ 0 , and (II) $q^{2} / R^{2}<0$, the $q^{2} / R^{2}=0$ case being a vertical line. These sectors are properly indicated in Figs 1 and 2 .

\section{On the imaginary electric charge}

From Eq. (37) the case of negative squared charge, $q^{2} / R^{2}<0$ also enters the analysis. It deserves some comments.

Equation (35) implies that for negative $q^{2}$ the parameter $a$ must be negative. This is clear after replacing $q^{2}$ by $-|q|^{2}$ and taking into account that $r_{0}^{2} / R^{2}$ is not larger than unity.

This has implications for the interior. From Eq. (18) one finds that in this case, i.e., for $a<0, Q^{2}(r)$ is negative. So $Q(r)$ is a pure imaginary number, as is $q$. The electric potential $\phi$ is also a pure imaginary function to guarantee the metric coefficient $B(r)$ is a real and positive function. On the other hand, all the metric spacetime functions are real since they depend on $q^{2}$ which is a real negative parameter.

The negativity of $q^{2}$ has also implications for the exterior. For negative $q^{2}$ the metric function of the external Reissner-Nordström spacetime, $B(r)=1 / A(r)=$ $1-2 m / r+q^{2} / r^{2}=1-2 m / r-|q|^{2} / r^{2}$, Eq. (25), has only one positive root, $r_{+}$, given by $r_{+}=m+\sqrt{m^{2}+|q|^{2}}$, i.e., it is given as in Eq. (32) with $q^{2} \rightarrow-|q|^{2}$. This is the gravitational radius of the solution, a horizon if $r_{0}<r_{+}$. Using Eq. (34) we can show that for $q^{2}<0$ the gravitational radius $r_{+}$is never larger than the radius of the matching boundary $r_{0}$, i.e., $r_{+} \leq r_{0}$, for $q^{2}<0$, with the equality holding in the limit $q^{2} \rightarrow-\infty$. Hence, no black hole-type solutions are found for $q^{2}<0$. Further properties of the solutions with $q^{2}<0$ are discussed later.

For a negative $q^{2}$ it is not possible to interpret $q$ as electric charge. Notwithstanding, a negative $q^{2}$ term was suggested by Einstein and Rosen in considering singularity free massless particles [4]. A similar situation happens for static spherical black hole solutions in a braneworld gravity scenario [44], where a tidal charge that may be negative plays the same role as the negative $q^{2}$ term in the Reissner-Nordström metric.

\section{B. Analysis of the mass parameter $m\left(q^{2}, r_{0}, R\right)$}

\section{Generics}

The behavior of the mass $m$ is given in Eq. (34). Clearly $m$ is a function of $q^{2}, r_{0}$ and $R$ (it is actually a function of $R^{2}$, but since $R>0$, the choice for $R$ or $R^{2}$ is indifferent, it is done here for reasons of better graphic displaying). Also from Eq. (34) one sees that the mass function $m\left(q^{2}, r_{0}, R\right)$ can be written formally as $m\left(q^{2}, r_{0}, R\right)=m\left(r_{0}, q^{2} / R^{2}, r_{0} / R\right)$. Figure 1 
shows the behavior of the mass function $m\left(q^{2}, r_{0}, R\right)=$ $m\left(r_{0}, q^{2} / R^{2}, r_{0} / R\right)$ given by Eq. (34). Figures 3 and 4 will also be referred to in this analysis.

To simplify the analysis, we consider separately the regions of the parameter space corresponding to five interesting classes of $m: m<0 ; m=0 ; 0<m<|q|$; $m=|q|$; and $m>|q|$, where again, for $q^{2}<0$ we denote $|q|=\sqrt{\left|q^{2}\right|}$ and for $q^{2}>0$ we denote $|q|=\sqrt{q^{2}}$. Of special interest is the class $m=|q|$, it is a curve that has two branches, namely, a closed curve in sector (I) and an open curve in sector (II), see Fig. 1.

\section{The various classes of $m$}

$$
m<0
$$

Since we are allowing mass contribution from the electromagnetic field to be negative, the total gravitational mass of the system can be negative. The related region of the parameter space is below the curve $m=0$ as seen in Fig. 1. In Figs. 3 and 4 the region corresponding to the $m<0$ solutions is labeled by $(\mathrm{h})$.

A particular case that can be dealt with analytically is the case $m=-|q|$. This solution is obtained by putting $-q^{2}=m^{2}$ into Eq. (34). The resulting negative mass is given by $m=r_{0}\left(-1-\sqrt{1+r_{0}^{2} / R^{2}}\right)$. The corresponding curve is not drawn in Fig. 1. The curve would be in region (h) of Figs. 3 and 4 but it is also not drawn.

All the solutions with $m<0$ are singular and seem to be of little physical interest.

$$
m=0
$$

The condition

$$
m\left(r_{0}, q^{2} / R^{2}, r_{0} / R\right)=0
$$

imposed to Eq. (34) furnishes the relation

$$
\frac{q^{2}}{R^{2}}=-\frac{r_{0}^{4}}{R^{4}}
$$

The Guilfoyle parameter is negative in this case, it is given by $a=-\left(r_{0}^{2} / R^{2}\right)\left(1-r_{0}^{2} / R^{2}\right)$. This yields the curve $m=0$ shown in sector (II) of Fig. 1, see also Figs. 3 and 4 where the curve $m=0$ is denoted by $C_{5}$. The solutions on this line present singularities and have little importance as star models.

$$
0<m<|q|
$$

The solutions with the mass $m$ in this range of values represent stars, see Fig. 1. The star radius $r_{0}$ is larger than the gravitational radius, i.e., $r_{0}>r_{+}$both when $q^{2}>0$ or $q^{2}<0$. For $q^{2}>0$ the stars are overcharged. Some of these solutions are regular, as those in regions (b) and (i) of Figs. 3 and 4, while others are singular, as those in regions (c) and (g) of the same figures. Notice that only parts of regions (g) and (i) contains object that satisfy $0<m<|q|$. The main aspects of these positive mass overcharged starts are examined in some detail later.

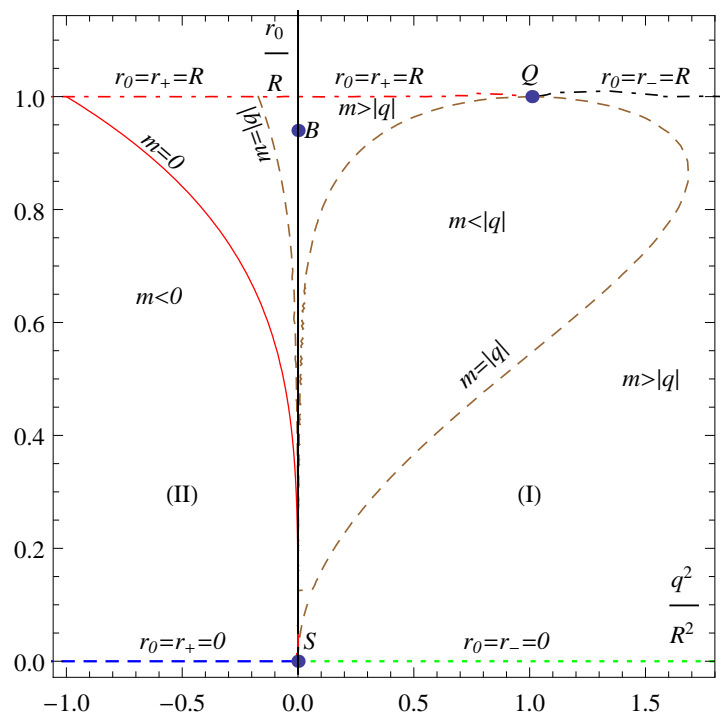

FIG. 1: Level curves for $m$ and corresponding areas in the $q^{2} / R^{2} \times r_{0} / R$ plane. For $q^{2} / R^{2}>0$ the curve for $m=|q|$ coincides with the curve for $a=1$. Also indicated are the lines $r_{0}=r_{-}$(dotted-dashed horizontal line $r_{0} / R=1$, with $\left.q^{2} / R^{2} \geq 1\right), r_{0}=r_{+}\left(\right.$dotted-dashed horizontal line $r_{0} / R=$ 1 , with $\left.q^{2} / R^{2} \leq 1\right), r_{+}=r_{0}=0$, and $r_{-}=r_{0}=0$, as well as the points $B$ representing the Buchdahl limit with $\left(q=0, m / r_{0}=4 / 9\right), Q$ representing the quasiblack hole limit with $\left(r_{+}=r_{-}=r_{0}=m=|q|\right)$, and $S$ representing the Schwarzschild black hole limit with $\left(q=0, r_{0}=0\right)$.

$$
m=|q|
$$

The condition

$$
m^{2}\left(r_{0}, q^{2} / R^{2}, r_{0} / R\right)= \pm q^{2}
$$

imposed to Eq. (34) yields two equations depending on the sign of $q^{2}$. We analyze the two cases separately.

$q^{2} / r_{0}^{2}<0$ : In this case Eqs. (34) and (41) give

$$
\frac{m^{2}}{r_{0}^{2}}+\frac{2 m}{r_{0}}-\frac{r_{0}^{2}}{R^{2}}=0 \text {. }
$$

There are two solutions to this equation, namely, $m=$ $r_{0}\left(-1 \pm \sqrt{1+r_{0}^{2} / R^{2}}\right)$. One of them yields a negative 
mass, which was already considered in the case $m<0$ above. Here we take the one that yields positive mass,

$$
m=|q|=r_{0}\left(-1+\sqrt{1+\frac{r_{0}^{2}}{R^{2}}}\right),
$$

or

$$
m^{2}=-q^{2}=r_{0}^{2}\left(2+\frac{r_{0}^{2}}{R^{2}}-2 \sqrt{1+\frac{r_{0}^{2}}{R^{2}}}\right) .
$$

This equation generates the line indicated by the appropriate label in sector (II) of Fig. 1. The solutions on this line can be regular stars or singular objects, and the pressure does not vanishes inside the fluid for these stars. The regular stars are located in region (i), while the singular solutions are located in region (g) of Figs. 3 and 4 , These solutions have not been studied in the literature. The region to the right-hand side of such a curve contains charged starlike solutions and other solutions with $m>|q|$, while the region to the left of that curve contains solutions with $m<|q|$.

$q^{2} / r_{0}^{2}>0$ : In this case Eqs. (34) and (41) give

$$
\frac{m^{2}}{r_{0}^{2}}-\frac{2 m}{r_{0}}+\frac{r_{0}^{2}}{R^{2}}=0 \text {. }
$$

The solution to this equation is

$$
m=|q|=r_{0}\left(1 \pm \sqrt{1-\frac{r_{0}^{2}}{R^{2}}}\right),
$$

or

$$
m^{2}=q^{2}=r_{0}^{2}\left(2-\frac{r_{0}^{2}}{R^{2}} \pm 2 \sqrt{1-\frac{r_{0}^{2}}{R^{2}}}\right) .
$$

The closed curve indicated by the label $m=|q|$ in the sector (I) of Fig. 11 is the complete solution, found joining the plus and minus solutions of Eq. (46). In Figs. 3 and 4 the $m=|q|$ solution is the sum of the curves denoted by $C_{0}$ and $C_{2}$. In the Guilfoyle's solutions [1] the condition $m=|q|$ is identical to the condition $a=1$. This means that for this class of solutions the pressure is zero everywhere inside the fluid [9] (see also [2]), corresponding to charged dust stars and also to quasiblack holes [3].

$$
m>|q|
$$

This class has objects for a large range of parameters and in several regions of the parameter space.

In the region $q^{2} / R^{2}<0$, sector (II) in Fig. 1, the solutions with $m>|q|$ are in the small portion of the sector between the axis $q^{2} / R^{2}=0$ and the line $m=|q|$. Objects in this region may be regular stars, as those in the part of region (i) in Figs. 3 and 4 above the curve $m=|q|$, or singular objects such those in the part of region (g) above the curve $m=|q|$.

In the region $0<q^{2} / R^{2}<1$, within sector (I) in Fig. 1, the objects with $m>|q|$ are locate in the region above the curve $m=|q|$. In Figs. 3 and 4 this region is labeled by (f) and (a). The Buchdahl-Andréasson bound divides the undercharged star solutions that are regular (in region (i)) from those that are singular (in region (f)). For the Buchdahl-Andréasson bound line see below and see curve $C_{4}$ in Figs. 3 and (4).

In the region $1<q^{2} / R^{2}<\infty$, within sector (I) in Fig. 1, the objects with $m>|q|$ are located are located in the region outside the curve $m=|q|$. Here the constraint $r_{0}<r_{-}$holds and the solutions are regular black holes, see [5] for more information. In Figs. 3 and 4 these solutions cover regions (d) and (e).

\section{Analysis of the parameter $a\left(q^{2}, r_{0}, R\right)$}

\section{Generics}

The behavior of the Guilfoyle parameter $a$ is given in Eq. (35). Clearly $a$ is a function of $q^{2}, r_{0}$ and $R$ (it is actually a function of $R^{2}$, but since $R>0$, the choice for $R$ or $R^{2}$ is indifferent, it is done here for reasons of better graphic displaying). Also from Eq. (35) one sees that the Guilfoyle parameter $a\left(q^{2}, r_{0}, R\right)$ can be written formally as $a\left(q^{2}, r_{0}, R\right)=a\left(q^{2} / R^{2}, r_{0} / R\right)$. Figure 2, where some important curves are drawn, shows the behavior of the Guilfoyle parameter $a\left(q^{2}, r_{0}, R\right)=a\left(q^{2} / R^{2}, r_{0} / R\right)$ given by Eq. (35). We identify some special regions in the parameter space according to the values of $a$ and give the general properties of the solutions belonging to each one of such regions. Figures 3 and 4 will also be referred in this analysis. Figure 1, which shows some curves for the mass $m$, is also useful in the study of the parameter $a$.

From Eq. (35), taking into account the parameter intervals given in Eqs. (37) and (38), it follows that $a$ assumes values in the interval

$$
-\infty<a<\infty .
$$

As seen from Eq. (35), the limit of infinite $a, a \rightarrow \infty$, corresponds to a vanishing electric charge. Also, from Eq. (6), in this limit the electric potential vanishes in such a way that the geometric function $B(r)$ remains finite. In this sense the Schwarzschild interior solution is a particular case of the Guilfoyle's solutions. The limit $a \rightarrow-\infty$ has the same properties as the limit $a \rightarrow \infty$. Moreover, $a$ also acquires arbitrarily large values when $r_{0} / R=0$ for $q^{2} \neq 0$, and $r_{0} / R=1$ for all values of 
$q^{2} / R^{2}$ except $q^{2} / r_{0}^{2}=1$. In the other limit, the limit of vanishing $a, a=0$, it requires that the electric charge assumes arbitrarily large values, in such a way that the limit of $a \phi^{2}$ is finite. This case is considered here for completeness. The parameter $a$ also vanishes for $q^{2} / R^{2}=$ $r_{0}^{4} / R^{4}$ with $r_{0}^{2} / R^{2} \neq 1$. Hence, $a=0$ is also of interest in the present study. The Guilfoyle parameter is therefore in the range given in Eq. (48).

Now it is convenient to consider separately the two sectors: (I) $q^{2} / R^{2}>0$ and $0 \leq r_{0} / R \leq 1$, where $a$ is positive; (II) $q^{2} / R^{2}<0$ and $0 \leq r_{0} / R \leq 1$, where $a$ is negative, as indicated in Fig. 2, see also Fig. 1. We draw some curves for constant values of $a\left(q^{2} / R^{2}, r_{0} / R\right)$ for the ranges given in Eqs. (37) and (38), i.e., $-\infty<q^{2} / R^{2}<$ $\infty$ and $0 \leq r_{0} / R \leq 1$, see Fig. 2 Considering that the $a=-\infty$ line closes at $q^{2} / R^{2} \rightarrow-\infty$, that $a=0$ line is a closed curve, actually trivially closed, and that the $a=-\infty$ line closes at $q^{2} / R^{2} \rightarrow \infty$, one finds that all contours $a=$ constant are closed curves.

To simplify the analysis, we study separately the regions of the parameter space in seven interesting classes of $a: a=-\infty ;-\infty<a<0 ; a=0 ; 0<a<1 ; a=1$; $1<a<\infty ; a=\infty$. Below we investigate each one of these classes.

\section{The various classes of a}

\section{$a=-\infty:$ Singular solutions \\ and uncharged Schwarzschild stars}

The limit $a=-\infty$ arises only for $q^{2} \leq 0$. In Fig. 2, the $a=-\infty$ limit is composed of the top horizontal line $r_{0} / R=1$ with $r_{0}=r_{+}=R$ in the sector $q^{2}<0$, plus the vertical line $q^{2} / r_{0}^{2}=0$, plus the bottom horizontal line $r_{0} / R=0$ with $r_{0}=r_{-}=0$ in the sector $q^{2}<0$. One can then think that the top and bottom horizontal lines join to form a closed curve at $q^{2} / R^{2} \rightarrow-\infty$. Indeed, the $a=-\infty$ limit appears in several instances that can be deduced using Eq. (35) as we now show.

One instance is when $r_{0} / R \rightarrow 1$. Here, one finds that the $a=-\infty$ limit admits charged singular solutions with $r_{0} / R=1$ and where $r_{0}=r_{+}=R$, see Fig. 2,

Another instance is for $q^{2} / r_{0}^{2} \rightarrow 0$ with nonzero $r_{0} / R$, i.e., the zero charged solutions with finite nonzero radius $r_{0}$ yield $a=-\infty$. These solutions compose the interior Schwarzschild solution and correspond to the vertical line $q^{2} / R^{2}=0$ in Fig. 2 .

Yet another instance is when $r_{0} / R \rightarrow 0$. Here, one finds that the $a=-\infty$ limit admits charged singular solutions where $r_{0}=r_{+}=0$, see Fig. 2.

Finally, one can then think that the top and bottom horizontal lines join at $q^{2} / R^{2} \rightarrow-\infty$ to form a closed

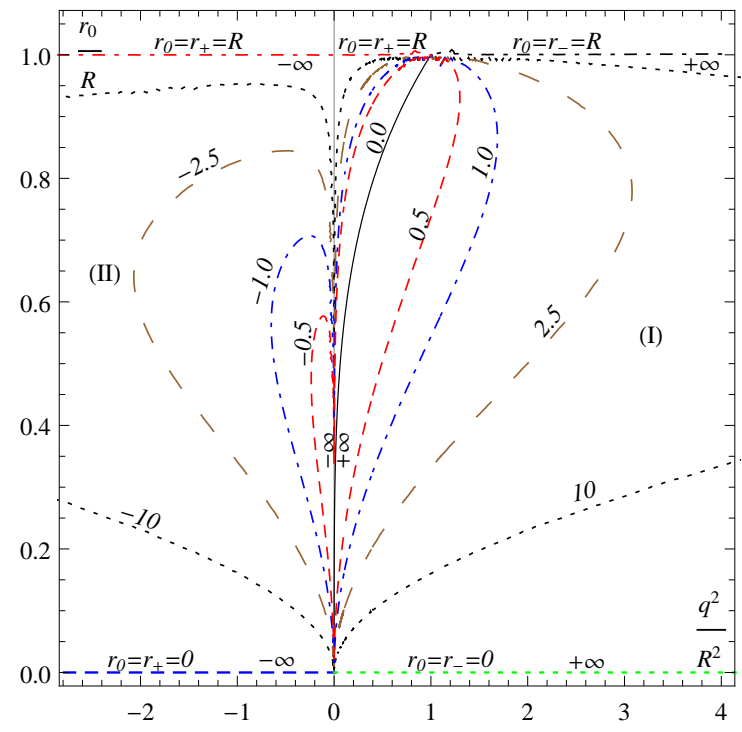

FIG. 2: Several contours for different values of the parameter $a$ as a function of the normalized charge $\left(q^{2} / R^{2}\right)$ and radius of the star $r_{0} / R$. The set of curves in the $q^{2}>0$ sector [sector (I)] are all for positive values of $a$, including $a=0$. The set of curves in the $q^{2}<0$ sector [sector (II)] are all for negative values of $a$. Curves with the following as are drawn: $a= \pm 0.0$ [solid line in sector (I) only], $a= \pm 0.5$ (dashed closed line, in both sectors), $a= \pm 1.0$ (dotted-dashed closed line, in both sectors), $a= \pm 2.5$ (spaced-dashed closed line, in both sectors), $a= \pm 10$ (dotted external curve, in both sectors). The $a= \pm \infty$ curves are composed of the three axes (top horizontal, vertical, bottom horizontal) in the $q^{2} \geq 0$ sector, and the three axes (top horizontal, vertical, bottom horizontal) in the $q^{2} \leq 0$ sector, respectively, and can be thought as closing at $q^{2} / R^{2} \rightarrow \pm \infty$, respectively. All contours are closed curves (the $a=0$ line can be considered a trivial closed curve). See text for details.

curve.

$-\infty<a<0$ : Regular stars and singular solutions

The negative $a$ class, $-\infty<a<0$, includes all the sector (II) in Fig. 2] which covers the parameter space portion given by $q^{2} / R^{2}<0$ (i.e., imaginary electric charge) and $0<r_{0} / R<1$. In Fig. 2 four representative curves of this class, namely, $a=-10, a=-2.5, a=-1.0$, and $a=-0.5$, are shown as a dotted closed line, a spaceddashed closed line, and a dotted-dashed closed line, and a dashed closed line, respectively.

In this class, the matching surface $r_{0}$ is outside the gravitational radius $r_{+}=m+\sqrt{m^{2}+|q|^{2}}$ (see Eq. (32) and the discussion in Sec. III A 2). So there are no black holes in this class. The solutions can be regular or singular, depending on the relative values of the parameters. A more complete discussion of sector (II) is given below, see also Figs. 3 and 4 
$a=0:$ The Schwarzschild vacuum solution, the quasiblack hole, and generic singular solutions

The curve

$$
a\left(r_{0}, q^{2} / R^{2}, r_{0} / R\right)=0,
$$

is shown in Fig. 2, it is the solid line in region (I). In Figs. 3 and 4 the curve is indicated by $C_{1}$. Considering the Guilfoyle relation, Eq. (6), one sees that Eq. (49), will give solutions with $B(r)=0$. Note also that from Eq. (35) the condition $a=0$ only occurs for $q^{2} / R^{2}>0$, i.e., $q^{2}>0$. Bearing in mind this restriction we can say the following for $a=0$.

From Eq. (35), one sees that Eq. (49), is satisfied in the plane $q^{2} / R^{2} \times r_{0} / R$, by the curve

$$
\frac{r_{0}}{R}=\sqrt{\frac{|q|}{R}},
$$

for $q^{2} / R^{2} \neq 0$ and $r_{0} / R \neq 0, q^{2} / R^{2} \neq 1$ and $r_{0} / R \neq 1$, and $q^{2} / R^{2}>0$ as stated above. The corresponding mass is $m=r_{0}^{3} / R^{2}=q^{2} / r_{0}$. The solutions in this curve represent singular solutions. They are without physical interest except for the two limiting end points of the curve.

The two limiting points of the curve, namely, $q^{2} / R^{2}=$ 0 and $r_{0} / R=0$, and $q^{2} / R^{2}=1$ and $r_{0} / R=1$, must be analyzed with care. In the case $q^{2} / R^{2}=0$ and $r_{0} / R=0$, one finds that to get $a=0$ the point must be approached in a specific way, namely, from Eq. (35) one should put first $r_{0}^{4}=R^{2} q^{2}$, and only then one takes $r_{0}^{2} / R^{2} \rightarrow 0$ and so $q^{2} / r_{0}^{2} \rightarrow 0$, then also $a=0$ (if one had put $q^{2} / r_{0}^{2}=$ 0 without $r_{0}^{4}=R^{2} q^{2}$ holding, then from Eq. (35) one gets $a \rightarrow \infty)$. This point is the point $S$ displayed in Figs. 3 and 4 and through this limit $S$ represents the Schwarzschild vacuum solution. The other possible case with Eq. (49) holding is the other limiting point $q^{2} / R^{2}=$ 1 and $r_{0} / R=1$. It is the quasiblack hole point $Q$ also displayed in Figs. 3 and 4 . These points will be discussed later on.

$$
\begin{gathered}
0<a<1: \text { Overcharged tension stars } \\
\text { and singular solutions }
\end{gathered}
$$

The class for which the condition $0<a<1$ holds occupies a portion in sector (I) of Fig. 2. It is also the same portion for which $m<|q|$, with $q^{2}>0$, see Fig. 1. In Fig. 2 one representative curve of this class, namely, $a=0.5$, is shown as a dashed closed line. In Figs. 3 and 4 such a portion covers the regions indicated by (b) and (c), and also the curve $C_{1}$. The matching surface is outside the gravitational radius, $r_{0}>r_{+}$, so the solutions in this portion are not black holes. For relatively large values of $r_{0}^{2} / q^{2}$, namely, for $r_{0}^{4}>q^{2} R^{2}$, within this portion, the solutions are regular everywhere, whereas for $r_{0}^{4}<$ $q^{2} R^{2}$, the solutions are singular in the sense that there are surfaces of infinite pressure or infinite energy density for some radius $r$ inside $r_{0}$. In Figs. 3 and 4, region (b) contains overcharged tension (negative pressure) stars, region (c) contains the singular solutions just mentioned.

$$
a=1: \text { Charged dust stars and other solutions }
$$

The equation

$$
a\left(q^{2} / R^{2}, r_{0} / R\right)=1,
$$

defines a closed curve indicated by a dotted-dashed line in sector (I) of Fig. 2, It is also the closed curve labeled as $m=|q|$ for $q^{2} / R^{2}$ in Fig. 1 . In Figs. 3 and 4 this closed curve is given by the union of the curves $C_{0}$ and $\mathrm{C}_{2}$.

All the solutions on this curve have zero pressure, $p(r)=0$, and the matter is extremely charged $\rho_{\mathrm{m}}=\rho_{\mathrm{e}}$, i.e., it is made of Majumdar-Papapetrou matter. For all these solutions, the spacetime region exterior to the boundary $r>r_{0}$ is the extreme Reissner-Nordström spacetime, for which $m=|q|$.

The left part of the $a=1$ curve in Fig. 2, labeled by $C_{0}$ in Figs. 3 and 4 , contains solutions that represent zero pressure regular charged stars. These are the Bonnor stars studied in the literature. The right part of the $a=1$ curve in Fig. 2, labeled by $C_{2}$ in Figs. 3 and 4 , contains solutions that represent zero pressure singular solutions, the energy density $\rho_{\mathrm{m}}$ reaching arbitrarily large values for certain values of the radius $r$ within the star.

The two parts of the $a=1$ curve meet at two points, the upper point $Q$ and the lower point $S$. The point $Q$ is the quasiblack hole limit (see Figs. 3 and 44). It is a degenerated point in the sense that, besides solutions with zero pressure, it contains solutions with nonzero pressure. $Q$ is the quasiblack hole point [3]. The point $S$ is also degenerated, it represents different solutions. The limit along these two parts of the curve gives the Minkowski empty spacetime. More details on these points are given later on.

$$
\begin{aligned}
& 1<a<\infty: \text { Charged stars, regular black holes, } \\
& \text { singular black holes, and other singular solutions }
\end{aligned}
$$

The class for which the condition $1<a<\infty$ holds occupies an outer portion in sector (I) of Fig. 2, In Fig. 2 two representative curves of this class, namely, $a=2.5$ and $a=10$, are shown as a spaced-dashed closed line and a dotted closed line, respectively.

There are several distinct subclasses of solutions. These subclasses appear distinctively in Figs. 3 and 4. and the corresponding regions are indicated by (a) which has undercharged stars studied in the original work by Guilfoyle [1], (d) which shows regular black holes with negative energy density [5], (e) which presents regular black holes with a central core of phantom matter studied 
in [5], and (f) which has singular solutions. A description of the properties of each class of solutions will be done later.

$a=\infty$ : Regular black holes with a de Sitter core, singular solutions, and uncharged Schwarzschild stars

The limit $a=\infty$ arises only for $q^{2} \geq 0$. In Fig. 2, the $a=\infty$ limit is composed of the top horizontal line $r_{0} / R=1$ with $r_{0}=r_{+}=R$ and $r_{0}=r_{-}=R$ in the sector $q^{2}>0$, plus the vertical line $q^{2} / r_{0}^{2}=0$, plus the bottom horizontal line $r_{0} / R=0$ with $r_{0}=r_{-}=0$ in the sector $q^{2}>0$. One can then think that the top and bottom horizontal line join to form a closed curve at $q^{2} / R^{2} \rightarrow \infty$. Indeed, the $a=\infty$ limit appears in several instances that can be deduced using Eq. (35) as we now show.

One instance is when $q^{2} / r_{0}^{2} \neq r_{0}^{2} / R^{2}$ and $r_{0} / R \rightarrow 1$. One finds that the $a=\infty$ limit admits charged solutions with $r_{0} / R=1$ for which the matching radius $r_{0}$ coincides with the inner horizon $r_{-}$of the Reissner-Nordström black hole 5] (see also [41]). These are regular black holes containing a perfect fluid of de Sitter type and a coat of electric charge at the surface boundary $r_{0}$, where $r_{0}=r_{-}=R$, see Fig. 2. One also finds that the $a=\infty$ limit admits charged singular solutions with $r_{0} / R=1$ and where $r_{0}=r_{+}=R$, see Fig. 2, Note that the excluded point in the analysis above, $q^{2} / R^{2}=1, r_{0} / R=1$, is the quasiblack hole point, indicated by $Q$ in Figs. 3 and 4. It is characterized by $r_{0}=r_{-}=r_{+}=R$. In this case $a\left(q, r_{0}\right)$ is not defined there, it has any value including $a=\infty$, depending on how each one of the parameters approaches unity. A more detailed analysis is given later.

Another instance is for $q^{2} / r_{0}^{2} \rightarrow 0$ with nonzero $r_{0} / R$, i.e., the zero charged solutions with finite nonzero radius $r_{0}$ yield $a=\infty$. These solutions compose the interior Schwarzschild solution and correspond to the vertical line $q^{2} / R^{2}=0$ in Fig. 2 .

Yet another instance is when $r_{0} / R \rightarrow 0$. Here, one finds that the $a=\infty$ limit admits charged singular solutions where $r_{0}=r_{-}=0$, see Fig. 2.

Finally, one can then think that the top and bottom horizontal lines join at $q^{2} / R^{2} \rightarrow \infty$ to form a closed curve.

\section{Special nontrivial cases that can be displayed analytically}

1. The Schwarzschild interior solution and the Schwarzschild star

In the limit of zero electrical charge $q^{2}=0$ one obtains expressions for the metric potentials and for the fluid quantities directly from the Guilfoyle's solution. In this limit $q^{2}=0$ one also has that the $a$ parameter obeys $a= \pm \infty$, see Eq. (35). Taking then the limit $q^{2} \rightarrow 0$ one gets for the functions given in Eqs. (11)-(16) the following expressions,

$$
\begin{gathered}
A(r)=\left(1-\frac{r^{2}}{R^{2}}\right)^{-1}, \\
B(r)=\frac{1}{4}\left(3 \sqrt{1-\frac{r_{0}^{2}}{R^{2}}}-\sqrt{1-\frac{r^{2}}{R^{2}}}\right)^{2}, \\
\phi \pi(r)=0, \\
8 \pi p(r)=-1+\frac{3}{2 \sqrt{1}-\frac{r^{2}}{R^{2}}}, \\
3 \sqrt{1-\frac{r_{0}^{2}}{R^{2}}-\sqrt{1-\frac{r^{2}}{R^{2}}}}, \\
\rho_{\mathrm{e}}(r)=0 .
\end{gathered}
$$

The functions $M(r)$ and $Q(r)$ of Eqs. (17) and (18) are now

$$
\begin{gathered}
M(r)=\frac{1}{2} \frac{r^{3}}{R^{2}}, \\
Q(r)=0
\end{gathered}
$$

respectively. The auxiliary function $F(r)$ given in Eq. (19) is still

$$
F(r)=k_{0} \sqrt{1-\frac{r^{2}}{R^{2}}}-k_{1},
$$

and $k_{0}$ and $k_{1}$ given in Eqs. (20) and (21) are now

$$
\begin{gathered}
k_{0}=\frac{m}{r_{0}} \frac{R^{2}}{r_{0}^{2}}, \\
k_{1}=\left(1+\frac{m}{r_{0}} \frac{R^{2}}{r_{0}^{2}}\right) \sqrt{1-\frac{r_{0}^{2}}{R^{2}}},
\end{gathered}
$$


respectively. The auxiliary function $F(r)$ and $k_{0}$ and $k_{1}$ are already embedded into Eqs. (52)-(57). Also the mass $m$ and the electric charge $q$ defined in Eqs. (22) and (23) are now

$$
\begin{gathered}
m=M\left(r_{0}\right)=\frac{1}{2} \frac{r_{0}^{3}}{R^{2}}, \\
q=Q\left(r_{0}\right)=0 .
\end{gathered}
$$

Of course this set of equations are the Schwarzschild interior solution. The exterior solution is the Schwarzschild spacetime, with the metric coefficients of Eqs. (24) and (25) being now given by

$$
\begin{gathered}
A(r)=\frac{1}{1-\frac{2 m}{r}}, \\
B(r)=\frac{1}{A(r)}=1-\frac{2 m}{r} .
\end{gathered}
$$

These metric potentials should then be inserted into the metric (1). The electric potential and the electric field components are equal to zero. Interior and exterior Schwarzschild solutions compose what one may call the Schwarzschild star.

Note that from Eq. (158) the mass $m$ of these Schwarzschild stars goes to zero with $r_{0}^{3}$. Therefore, the resulting solution in the limit of $r_{0}=0$ is the Minkowski spacetime.

In Figs. 1.4 the Schwarzschild stars are contained in the line segment $q^{2} / R^{2}=0$ and $0<r_{0} / R<1$. When one takes the limit of the line $q^{2} / R^{2}=0$ down to $r_{0} / R=0$ one arrives at the point $S$ shown in Fig. 1 1 and Figs. 3, 4 , which in this limit represents Minkowski spacetime. In Fig. 2 the line $q^{2} / R^{2}=0$ and $0<r_{0} / R<1$ is part of the curves $a \rightarrow \pm \infty$.

\section{The Buchdahl-Andréasson bound}

An important relation between the parameters of the solutions, i.e., between $m, q$, and $r_{0}$, appears when the fluid pressure at the center of the star goes to infinity. This limit gives the Buchdahl-Andréasson bound [4],

$$
\frac{m}{r_{0}}=\left(\frac{1}{3}+\sqrt{\frac{1}{9}+\frac{q^{2}}{3 r_{0}^{2}}}\right)^{2} .
$$

From Eq. (67) one can extract three interesting cases. For $q^{2} / r_{0}^{2}=0$, i.e., the zero electrically charged case, one gets the Buchdahl bound, $m / r_{0}=4 / 9$ [4]. For $q^{2} / r_{0}^{2}=1$, i.e., the extremal case, one gets the quasiblack hole limit, $m / r_{0}=1$ [3]. For $q^{2} / r^{2}=-1 / 3$, i.e., the maximal compact star, one gets the maximum value of the Buchdahl-Andréasson bound, $m / r_{0}=9$, indeed a very compact star that has negative $q^{2}$, not considered in [4], but clearly the bound also holds for negative $q^{2}$ as long as one has $q^{2} / r_{0}^{2} \geq-1 / 3$.

The Buchdahl-Andréasson bound appears as the curve $C_{4}$ in Figs. 3 and 4 .

\section{The case $r_{0}=r_{+}$}

When the radius of the object $r_{0}$ reaches $r_{+}$one has two situations. From Eq. (32) one has $r_{0}=m\left(q, r_{0}, R\right)+$ $\sqrt{m^{2}\left(q, r_{0}, R\right)-q^{2}}$ and from Eq. (34) this yields

$$
r_{0}=\frac{q^{2}}{2 r_{0}}+\frac{r_{0}^{3}}{2 R^{2}}+\sqrt{\left(\frac{q^{2}}{2 r_{0}}+\frac{r_{0}^{3}}{2 R^{2}}\right)^{2}-q^{2}} .
$$

Equation (68) has two solutions.

One solution is

$$
r_{0}=r_{+}=R,
$$

which is satisfied as long as $-\infty<q^{2} / R^{2}<1$. It represents infinite pressure solutions with matching at the Reissner-Nordström horizon, i.e., singular solutions.

The other solution is

$$
r_{0}=r_{+}=0,
$$

satisfied for all $q^{2} \leq 0$. It represents negative mass naked singularities, see Appendix A 1 for details.

In Figs. 1, 4 these two sets of singular solutions are represented and explicitly indicated by the appropriate labels. In Fig. 2 the solutions mentioned in this section are part of the curves $a= \pm \infty$.

\section{The case $r_{0}=r_{-}$}

When the radius of the object $r_{0}$ reaches $r_{-}$one has very interesting situations. From Eq. (33) one has $r_{0}=$ $m\left(q, r_{0}, R\right)-\sqrt{m^{2}\left(q, r_{0}, R\right)-q^{2}}$ and from Eq. (34) this yields

$$
r_{0}=\frac{q^{2}}{2 r_{0}}+\frac{r_{0}^{3}}{2 R^{2}}-\sqrt{\left(\frac{q^{2}}{2 r_{0}}+\frac{r_{0}^{3}}{2 R^{2}}\right)^{2}-q^{2}} .
$$

Equation (71) has two solutions. 
One solution is

$$
r_{0}=r_{-}=R \text {, }
$$

which is satisfied only for $q^{2} / R^{2}>1$. It gives regular black holes with a de Sitter core with a matching at the Cauchy horizon. These solutions were studied in [5] (see also [41]).

The other solution is

$$
r_{0}=r_{-}=0 \text {, }
$$

satisfied for all $q^{2} / R^{2}>0$. It is a Kasner spacetime time, see Appendix A2 for details.

In Figs. 1, 4 these two sets of solutions are represented and explicitly indicated by the appropriate labels. In Fig. 2 the solutions mentioned above are part of the curves $a= \pm \infty$.

\section{The case $r_{0}=r_{+}=r_{-}$}

When the radius of the object $r_{0}$ reaches a double horizon $r_{+}=r_{-}$one has from Eqs. (32)-(34) the result

$$
r_{0}=r_{+}=r_{-}=m=q=R .
$$

This is the quasiblack hole. It is an extremal object with very interesting properties, for details see [3].

In Figs. 1, 4 the quasiblack hole is represented. In Fig. 1 and Figs. 30 4 it is explicitly indicated by the appropriate labels. In Fig. 2 it is a point in all positive $a$ curves, in particular in the curve $a=\infty$.

\section{E. Generics to the next two sections}

A point in the parameter space $q^{2} / R^{2} \times r_{0} / R$ represents one solution. Thus all solutions are represented in this parameter space. These solutions can be physical or unphysical, although this division can be subjective. In the sector $q^{2} \geq 0$, the physical ones are normal undercharged stars, dust extremely charged stars, overcharged tension stars, and regular black holes with a phantom matter core, and the unphysical ones are regular black holes with negative energy densities and singular solutions. In the sector $q^{2}<0$, there are regular and singular solutions.

Figures 3 and 4 display the regions, areas in the figure, where the solutions are regular, represented by white regions, and solutions where the pressure and, or, the energy-density inside the fluid distribution are singular, represented by gray regions. The boundaries of these regions are lines or points which also represent solutions.
In the next two sections we comment carefully what the regions and boundaries represent physically. We start with regions in the $q^{2} / R^{2} \geq 0$ sector [regions (a), (b), (c), (d), (e), and (f)], some of them have solutions with interesting physical meaning, and then continue into regions in the $q^{2} / R^{2}<0$ sector [regions $(\mathrm{g}),(\mathrm{h})$, and (i)]. We also study the lines and points, boundaries of the analyzed areas, namely, the curves $C_{0}, C_{1}, C_{2}, C_{3}, C_{4}, C_{5}$, the horizontal and vertical axes and lines, and the points $S, B$, and $Q$.

The analysis has been performed using Mathematica and exploring in detail the whole spectrum of this plethora of solutions. It was a meticulous work.

\section{REGULAR AND SINGULAR REGIONS (AREAS) IN THE PARAMETER SPACE $q^{2} / R^{2} \times r_{0} / R$}

\section{A. Region (a): Undercharged stars}

Region (a) is the region of undercharged nonsingular stars and is delimited by three curves, namely, the $q^{2} / R^{2}=0$ coordinate axis from the origin up to the point $B$, the sector of curve $C_{4}$ joining $B$ to the point $Q$ (separating gray and white regions), and the curve of zero pressure indicated by $C_{0}$, see Figs. 3 and $4 . C_{0}$ is a branch of the curve $m=|q|$, or $a=1$, and $C_{4}$ is the Buchdahl-Andréasson curve, see also Figs. 1 and 2.

Some more detail is now given. Undercharged stars are characterized by $m>|q|$. In this nonsingular region of undercharged stars, region (a), the constraint $r_{0} / r_{+}>1$ is satisfied, so that black holes are not present. Moreover, the Guilfoyle parameter $a$ satisfies the constraint $a>1$ in the whole region, except at the boundaries. The stars with radius $r_{0}$ and charge $q$ belonging to this region of the parameter space satisfies the energy conditions, as studied by Guilfoyle 1]. Further analysis of the objects, including the point $Q$ in Figs. 3] and 4, is given in [2].

The boundaries of region (a) have solutions whose physical properties are discussed later.

\section{B. Region (b): Overcharged tension stars}

Region (b) is the region of overcharged nonsingular tension stars and is delimited by $C_{0}$ and $C_{1}$ in Figs. 3 and 4. $C_{0}$ is a branch of the curve $m=|q|$, or $a=1$, and $C_{1}$ is the curve given by $a=0$, see also Figs. 1 and 2

Some more detail is now given. Overcharged stars are characterized by $m<|q|$. The parameter space for 


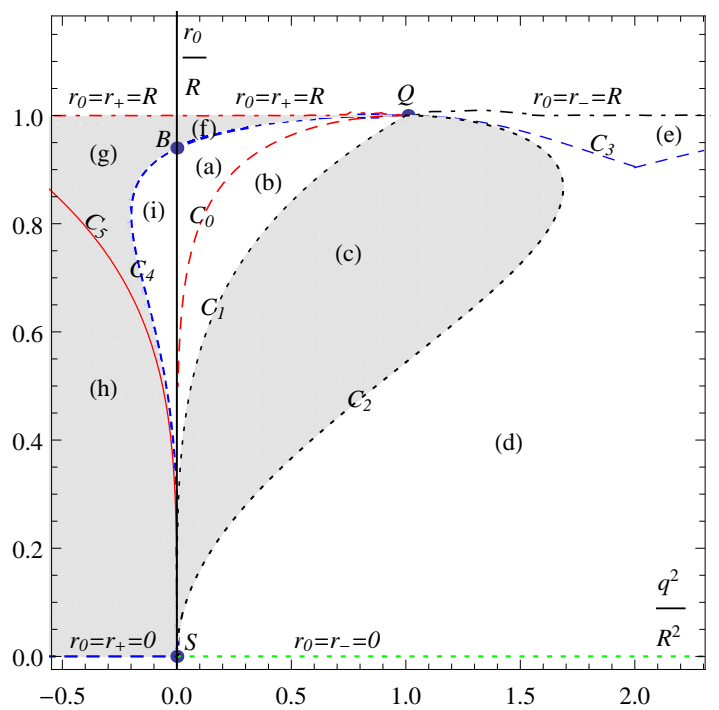

FIG. 3: The white regions contain regular solutions and the gray regions contain singular solutions. The boundaries between two regular regions contain regular solutions. The boundaries between a regular region and a singular one, or between two singular regions, contain singular solutions.

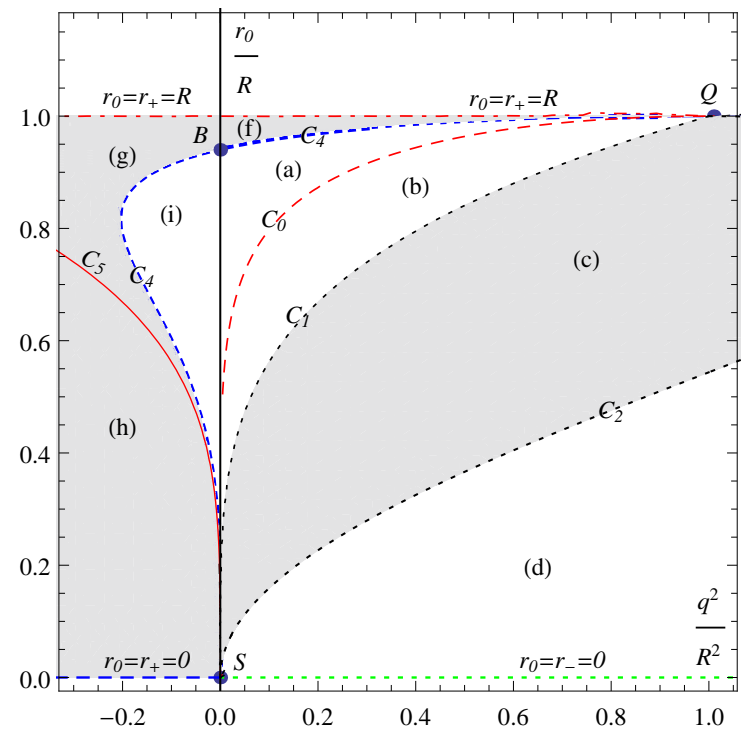

FIG. 4: The same as Fig. 3 zoomed in. Regions with regular (white) and singular (gray) solutions and their boundaries are indicated.

the whole set, singular and nonsingular overcharged solutions, with $m<|q|$ can be found using Eq. (34). The condition $m \leq|q|$ gives $|q|^{2} / r_{0}^{2}-2|q| / r_{0}+r_{0}^{2} / R^{2} \leq 0$, see also Eq. (45). The equality furnishes the curve $m=|q|$ which for $q^{2} / R^{2}>0$, i.e., $q^{2}>0$, is the closed curve formed by the $C_{0}$ and $C_{2}$ curves in Figs. 3 and 4 . Thus, the overcharged region of the parameter space is the whole region inside such a curve. The curve $C_{1}$ separates the nonsingular from the singular overcharged solutions. In- deed, in the $C_{1}$ line the Guilfoyle parameter vanishes, $a\left(q^{2}, r_{0}\right)=0$ [see Eq. (50)] and the fluid quantities are singular. So, nonsingular overcharged solutions are delimited by $C_{0}$ and $C_{1}$.

Note that the curve $m=|q|$ in the sector $q^{2} / R^{2}>0$ is the closed curve explicitly given in Fig. 1. Since $m=$ $|q|$ coincides with the $a=1$ curve, one also finds that the overcharged region corresponds to small values of the Guilfoyle parameter $a$, namely, $0<a<1$, and that in these solutions the pressure within the fluid is negative $p(r)<0$ for all solutions there, i.e., it is a tension.

The boundaries of region (b) have solutions whose physical properties are discussed later.

\section{Region (c): Overcharged solutions, wildly behaved solutions}

Region (c) is the region of overcharged singular solutions with wild behavior of the energy density and pressure and is delimited by the curves $C_{1}$ and $C_{2}$, see Figs. 3 and $4 . C_{1}$ is the curve given by $a=0$, and $C_{2}$ is a branch of the curve $m=|q|$, or $a=1$, see also Figs. 1 and 2,

Some more detail is now given. These are overcharged solutions still with $m<|q|$. The energy density of the solutions in this region behaves wildly, it is positive and finite at the center and changes to very high negative values close to the surface. The pressure of these solutions behaves as wildly as the energy density, it is negative and finite at the center, and oscillates to very high positive values close to the surface of the solution and finally goes to zero at the surface. Because of this wild behavior, these solutions are highly weird and we do not study them further.

The boundaries of region (c) have solutions whose physical properties are discussed later.

\section{Region (d): Regular black holes with negative energy densities}

Region (d) is the region of regular black holes with negative energy delimited by the curves $C_{2}$ and $C_{3}$, and the horizontal line $r_{0}=r_{-}=0$ in Fig. 3 part of it being seen also in Fig. 4. $C_{2}$ is a branch of the curve $m=|q|$, or $a=1, C_{3}$ is a curve with no special features in terms of the parameters $m$ or $a$, and the horizontal line $r_{0}=r_{-}=0$ is a portion of the curve $a=\infty$, see also Figs. 1 and 2 ,

Some more detail is now given. The solutions in region (d) are characterized by several facts. First, the matching 
surface defined by $r_{0}$ is timelike, and its radius is smaller than the Cauchy horizon radius $r_{-}, r_{0} / r_{-}<1$. So the solutions represent black holes. Second, the solutions are regular, i.e., there are no singularities. Third, the energy density is negative for a range of radii in the solutions and the pressure can also be negative going to zero at the surface $r_{0}$. Fourth, due to the negativity of the energy density, these regular black holes have less interest than those of region (e) below.

The boundaries of region (d) have solutions whose physical properties are discussed later.

\section{E. Region (e): Regular black holes with a phantom matter core}

Region (e) is the region of regular black holes with a phantom matter core and is delimited by the curve $C_{3}$ and the line $r_{0}=r_{-}=R$ in Fig. 3, it is outside the range of Fig. 4. $C_{3}$ is a curve with no special features in terms of the parameters $m$ or $a$, the line $r_{0}=r_{-}=R$ is a branch of the $a=\infty$ curve, see also Figs. 1] and 2,

Some more detail is now given. The solutions in region (e), like those in region (d) above, are characterized by several facts. First, the matching surface defined by $r_{0}$ is timelike, and its radius is smaller than the Cauchy horizon radius $r_{-}, r_{0} / r_{-}<1$. So the solutions represent black holes. Second, the solutions are regular, i.e., there are no singularities. Third, the energy density is positive everywhere inside matter, the pressure is negative and it goes to zero at the surface $r_{0}$. Fourth, this negative pressure at the center is larger than the central density, thus for a finite region inside the matter one finds $\rho_{\mathrm{m}}+p<0$, meaning there is phantom matter and consequently the energy conditions are violated. In brief, in region (e) one gets regular black holes with an electrically charged phantom matter core. These regular black holes were investigated in [5].

The boundaries of region (e) have solutions whose physical properties are discussed later.

\section{F. Region (f): Singular solutions}

Region (f) is a region of singular solutions, delimited by three curves, namely, the $q^{2} / R^{2}=0$ coordinate axis from the point $B$ up to $r_{0} / R=1$, by the curve $C_{4}$ in the $q^{2} / R^{2}>0$ sector, and by the line $r_{0}=r_{+}=R$, see Figs. 3 and 4. The curve $C_{4}$ is the Buchdahl-Andréasson bound curve, and the vertical line $q^{2} / R^{2}=0$ and the horizontal line $r_{0}=r_{+}=R$ are part of the $a=\infty$ curve, see also Figs. 1 and 2 .
All solutions in region (f) are singular, since the pressure diverges at some point inside the matter distribution.

The boundaries of region (f) have solutions whose physical properties are discussed later.

\section{G. Region (g): Singular solutions with $m>0$ and $q^{2}<0$}

Region (g) is the region of singular solutions with $m>$ 0 and $q^{2} / R^{2}<0$, i.e., $q^{2}<0$, and is delimited by the $q^{2} / R^{2}=0$ coordinate axis from the point $B$ up to $r_{0} / R=$ 1 , the horizontal line $r_{0}=r_{+}=R$, the curve $C_{5}$, and the curves $C_{4}$ in the sector $q^{2} / R^{2}<0$, see Fig. 3 and in more detail Fig. 4. The curve $C_{4}$ is the BuchdahlAndréasson bound curve, the vertical line $q^{2} / R^{2}=0$ and the horizontal line $r_{0}=r_{+}=R$ are a portion of the curve $a=-\infty$, and the curve $C_{5}$ is the curve $m=0$, see also Figs. 1 and 2 .

For all solutions in this region of the parameter space the energy density becomes infinitely large and the pressure becomes arbitrarily large and negative for some radius inside the matter distribution. Thus, this region of the parameter space bears solutions of limited physical interest.

The boundaries of region (g) have solutions whose physical properties are discussed later.

\section{H. Region (h): Singular solutions with $m<0$ and $q^{2}<0$}

Region (h) is the region of singular solutions with negative mass, $m<0$, which implies here $q^{2} / R^{2}<0$, i.e., $q^{2}<0$, and is delimited by the curve $C_{5}$ and the horizontal line $r_{0}=r_{+}=0$, see Fig. 3 and in more detail Fig. 4 . The curve $C_{5}$ is the curve $m=0$ and the horizontal line $r_{0}=r_{+}=0$ is a portion of the curve $a=-\infty$, see also Figs. 1] and 2 .

This region presents solutions which are similar to those belonging to region $(\mathrm{g})$, the only difference is that here the mass is negative, $m<0$. The solutions are singular, the matter interior is plagued with energy densities arbitrarily large, and the pressure also assumes arbitrarily large negative values. These singular solutions have also limited interest.

The boundaries of region (h) have solutions whose physical properties are discussed later. 
I. Region (i): Regular stars with $m>0$ and $q^{2}<0$

Region (i) is the region of regular stars with positive mass, $m>0$, and $q^{2} / R^{2}<0$, i.e., $q^{2}<0$, and is delimited by the $q^{2} / R^{2}=0$ coordinate axis from the origin up to the point $B$, and a portion of the curve $C_{4}$ in the sector $q^{2} / R^{2}<0$, see Figs. 3 and 4 , The vertical line $q^{2} / R^{2}=0$ is part of the curve $a=-\infty$ and the curve $C_{4}$ is the Buchdahl-Andréasson bound curve, see also Figs. 1 and 2 .

Some more detail is now given. In this region since $q^{2}<0$ the electric charge $q$ is imaginary, and the Guilfoyle parameter $a$ is negative, $a<0$. One finds that the radius $r_{0}$ of the matching surface is greater than the gravitational radius, $r_{0}>r_{+}$where $r_{+}=m+\sqrt{m^{2}+|q|^{2}}$. The solutions are nonsingular. So the solutions represent charged stars with imaginary electric charge.

The boundaries of region (i) have solutions whose physical properties are discussed later.

\section{REGULAR AND SINGULAR BOUNDARIES (LINES AND POINTS) IN THE PARAMETER SPACE $r_{0} / R \times q^{2} / R^{2}$}

\section{A. Curve $C_{0}$ : Bonnor stars}

The curve $C_{0}$ is the line of charged dust stars. It begins at point $S$ and extends to point $Q$, see Figs. 3 and $4 . C_{0}$ corresponds to $m=|q|$ in the sector $q^{2}>0$ and is part of the closed $a=1$ curve, see also Figs. 1 and 2

On $C_{0}$ the fluid pressure vanishes throughout the interior of the matter distribution, so it is dust, and the spacetime region exterior to the boundary $r>r_{0}$ is the extreme $m=|q|$ Reissner-Nordström spacetime. The stars in this curve are called Bonnor stars, i.e., charged dust stars [10, 11].

\section{B. Curve $C_{1}$ : Boundary of regular overcharged tension star solutions}

The curve $C_{1}$ has overcharged solutions and it separates region (b) of regular overcharged stars with tension from region (c) of overcharged singular solutions with tension, see Figs. 3 and [4. $C_{1}$ has objects with $m<|q|$ in the sector $q^{2}>0$ and it corresponds to $a=0$, i.e., it is the curve given by the equation $q^{2} / R^{2}=r_{0}^{4} / R^{4}$, see Eq. (50), see also Figs. 1 and 2 .

On $C_{1}$ the solutions are singular.

\section{Curve $C_{2}$ : Singular charged dust solutions}

The curve $C_{2}$ is the line of singular charged dust solutions, and as $C_{0}$ it begins at point $S$ and extends to point $Q$, see Figs. 3 and $4 . C_{2}$ also corresponds to $m=|q|$ in the sector $q^{2}>0$ and is the other part of the closed $a=1$ curve, see also Figs. 1 and 2 .

On $C_{2}$ the solutions contain matter that is extremely charged, the pressure is zero, however, the energy density is negative assuming very large values close to the center of the solution, a similar behavior to the solutions of region (c). We regard these solutions as singular solutions.

D. Curve $C_{3}$ : Boundary between regular black holes with phantom matter and regular black holes with negative energy matter

The curve $C_{3}$ is the line that divides regular black holes with negative energy matter shown in region (d) from regular black holes with phantom matter shown in region (e), see Figs. 3 and 4 . The curve $C_{3}$ has no relation with the $m$ and $a$ curves displayed in Figs. 1 and 2 ,

For the regular black holes on $C_{3}$, the matching surface radius $r_{0}$ is smaller than the Cauchy horizon of the Reissner-Nordström metric $r_{-}, r_{0}<r_{-}$. Even though being regular, the matter functions inside $r_{0}$ present some peculiar properties, for instance, the energy density vanishes for some radii $r$.

\section{E. Curve $C_{4}$ : The Buchdahl-Andréasson bound}

The curve $C_{4}$ is the Buchdahl-Andréasson bound line, i.e., it is the line obtained taking the limit of the central pressure going to infinity, see Figs. 3 and 4 . The curve $C_{4}$ has no relation with the $m$ and $a$ curves displayed in Figs. 1 and 2

The Buchdahl-Andréasson bound is discussed in Sec. ПIID 2, particularly Eq. (67). The BuchdahlAndréasson bound penetrates the region of negative electrical charge, $q^{2}<0$, a region not considered in [4]. The bound holds as long as one has $q^{2} / r_{0}^{2} \geq-1 / 3$. The maximum value of the Buchdahl-Andréasson bound is $m / r_{0}=9$ for $q^{2} / r_{0}^{2}=-1 / 3$, a very compact star with negative $q^{2}$. The curve $C_{4}$ contains the three very special and important points, the quasiblack hole point $Q\left(q^{2} / R^{2}=1, r_{0} / R=1\right)$, the Buchdahl point $B\left(q^{2} / R^{2}=0, r_{0} / R=2 \sqrt{2} / 3\right)$, and the Schwarzschild point $S\left(q^{2} / R^{2}=0, r_{0} / R=0\right)$. 


\section{F. Line $r_{0}=r_{+}=R$ and $0<q^{2}<R^{2}$ : Singular solutions with the matter boundary at the Reissner-Nordström gravitational radius}

The line $r_{0} / R=r_{+} / R=1$ for $0<q^{2} / R^{2}<1$ is a line that represents singular solutions with the matter boundary at the Reissner-Nordström gravitational radius $r_{0} / r_{+}=1$, it starts at $q^{2} / R^{2}=0$ and goes up to point $Q$, see Figs. 3and 4 . This line has no relation with the $m$ curves and is a part of the $a=\infty$ curve, see also Figs. 1 and 2 .

Each point on this line represents a solution where the interior region, i.e., from $r=0$ up to $r=r_{0}=r_{+}$, is filled with a charged fluid, and it is matched to the exterior Reissner-Nordström region by a lightlike surface. However, this matching can be realized only for infinite pressure, and thus the solutions are not well defined, they are singular. The line is discussed in Sec. IIID3

G. Line $r_{0}=r_{-}=R$ and $q^{2}>R^{2}$ : Regular black holes with a de Sitter core and the matter boundary at the Cauchy horizon

The line $r_{0} / R=r_{-} / R=1$ for $q^{2} / R^{2}>1$ is a line that represents regular black holes with a de Sitter core and the matter boundary at the Cauchy horizon $r_{0} / r_{-}=1$, it starts at point $Q$ and goes up to infinity to the right, see Figs. 3 and 4 This line has no relation with the $m$ curves and is a part of the $a=\infty$ curve, see also Figs. 1 and 2 .

Each point on this line represents a regular charged black hole whose interior region is de Sitter, the exterior is Reissner-Nordström, and the matching surface is a layer of uniform charge density, the total charge being located on the boundary surface, i.e., at the Cauchy horizon. These solutions were studied in detail in [5] (see also [4]). The line is discussed in Sec. IIID4.

\section{H. Line $r_{0}=r_{-}=0$ and $q^{2}>0$ : Kasner spacetimes}

The line $r_{0} / R=r_{-} / R=0$ for $q^{2} / R^{2}>0$ is a line for which each point represents a Kasner spacetime, see Figs. 3 and 4, This line has no relation with the $m$ curves and is a part of the $a=\infty$ curve, see also Figs. 1 and 2 .

The Kasner spacetime has planar symmetry and appears in several contexts, see Appendix A2.

\section{Line $0<r_{0}<R$ and $q^{2}=0$ : The Schwarzschild star}

The line $0<r_{0} / R<1$ for $q^{2} / R^{2}=0$ is a line that represents the whole class of neutral, i.e., electrically uncharged, solutions within the Guilfoyle's solutions [1]. It starts at the point $S$ and goes up vertically up to the point $r_{0} / R=1$, see Figs. 3 and 4 . This line has no relation with the $m$ curves and is a part of both the $a=\infty$ and the $a=-\infty$ curves, see also Figs. 1 and 2 .

Each point on this line represents a Schwarzschild star, i.e., the Schwarzschild interior solution matched to the Schwarzschild exterior solution, see Sec. IIID 1 Note that from Eq. (58) the mass of these stars goes to zero as $r_{0}^{3}$. Therefore, the resulting solution found by taking the limit to the point $S$ down the line $q^{2} / R^{2}=0$ is the Minkowski spacetime, see Figs. 3 and 4 .

\section{J. Curve $C_{5}$ : Singular zero mass solutions}

The curve $C_{5}$ represents zero mass solutions which can only exist in these solutions for $q^{2}<0$, see Figs. 3 and 4 , It also appears in Fig. 1 as the curve $m=0$ in the $q^{2}<0$ sector, in sector (I). This line has no relation with the $a$ curves, see Fig. 2 .

The Guilfoyle parameter $a$ is negative for these solutions. The fluid quantities in this curve are not well defined and the solutions are of no physical interest. See also Sec. IIIB 2, particularly Eq. (40).

\section{K. Line $r_{0}=r_{+}=R$ and $q^{2}<0$ : Singular solutions with the matter boundary at the \\ Reissner-Nordström gravitational radius in the negative electric charge square sector}

The line $r_{0} / R=r_{+} / R=1$ for $q^{2} / R^{2}<0$ is a line that represents singular solutions with the matter boundary at the Reissner-Nordström gravitational radius, it starts at $q^{2} / R^{2}=-\infty$ and goes up to point $q^{2} / R^{2}=0$, see Figs. 3 and 4 This line has no relation with the $m$ curves, see Fig. 1, and is a part of the $a=-\infty$ curve, see Fig. 2,

Each point on the line $r_{0}=r_{+}=R, q^{2}<0$ represents a solution where the interior region, i.e., from $r=0$ up to $r=r_{0}=r_{+}$, is filled with a charged fluid with negative square charge, and it is matched to the exterior ReissnerNordström region by a lightlike surface. However, this matching can be realized only for infinite pressure, and thus the solutions are not well defined they are singular. These solutions have similar behavior to those with $r_{0}=$ $r_{+}=R, 0<q^{2}<R^{2}$ discussed in Sec. $\mathrm{VF}$ The line is 
also discussed in Sec. IIID 3.

\section{Line $r_{0}=r_{+}=0$ and $q^{2}<0$ : Naked singularities in the negative electric charge square sector}

The line $r_{0} / R=r_{+} / R=0$ for $q^{2} / R^{2}<0$ is a line that represents naked singularities, see Figs. 3 and 4 . This line has no relation with the $m$ curves, see Fig. 1, and is a part of the $a=-\infty$ curve, see Fig. 2,

In this case the spacetime for small $r$ has planar symmetry and is singular at $r=0$, see Sec. IIID 3, It represents naked singularities, see also Appendix A1

\section{Point S, $q^{2}=0, r_{0}=0$ : The Minkowski spacetime, the Kasner solution, the Schwarzschild solution, the Schwarzschild negative mass singularity, and the plane-symmetric static spacetime naked singularity}

\section{Point $S$ from above, $q^{2}=0, r_{0} \rightarrow 0$ : Minkowski spacetime}

When one approaches point $S$ from above, i.e., performing $q^{2} / R^{2}=0$ and $r_{0} / R \rightarrow 0$, see Figs. 3 and 4 , one finds a Minkowski spacetime.

In this limit the mass $m$ indeed vanishes and the result is the Minkowski spacetime as follows from Eq. (58) and we have commented in Sec. VI In addition, in this limit the parameter $a$ becomes arbitrarily large.

2. Point $S$ from the right, $r_{0}=r_{-}=0, q^{2} \rightarrow 0^{+}:$Kasner spacetime

When one approaches point $S$ from the right, i.e., performing $r_{0} / R=r_{-} / R=0$ and $q^{2} / R^{2} \rightarrow 0^{+}$, see Figs. 3 and 4, one finds a Kasner spacetime.

The limiting process is to take $r_{0} / R$ to zero, independently of $q^{2} / R^{2}$, and then taking $q^{2} / R^{2}$ to zero. The first part of the process leads to the Kasner metric, and the second part of the process does not change the resulting geometry, because the charge and mass are transformed way by changing the coordinates. The result is then a Kasner spacetime, see also Sec. $\mathrm{VH}$ and Appendix $\mathrm{A} 2$. In this limit $a=\infty$.
3. Point $S$ from the skew right, $r_{0}>0, q^{2}>0$ : Minkowski spacetime, Schwarzschild black hole, and Kasner spacetime

\section{Generics}

When one approaches point $S$ from a skew path on the right, see Figs. 3 and 4 , one finds, depending on the approaching path, a Minkowski spacetime, or a Schwarzschild black hole, or a Kasner spacetime. All the three cases have zero electric charge and zero electric potential, of course, since point $S$ has $q^{2}=0$. Some physical quantities on $S$ have values that depend on how the point is approached. A particular important quantity is the mass. That is a reason to get different spacetimes on approaching $S$ by different paths.

To see this, let $\gamma$ be a path in the two-dimensional parameter space spanned by the parameters $q^{2} / R^{2}$ and $r_{0} / R$ approaching $S$. The curve $\gamma$ may be parametrized by $q^{2} / R^{2}$ so that along $\gamma$ we may write $r_{0} / R=f\left(q^{2} / R^{2}\right)$. To simplify the notation from now onward define $x \equiv$ $q^{2} / R^{2}$ and $y \equiv r_{0} / R$, so that the two-dimensional space is $x \times y$ and along $\gamma$ we may write $y=f(x)$ with $\gamma$ being parametrized by $x$. Assume $f$ to be a piecewise continuous function of its argument. Since $f(x)$ has also to satisfy the condition $\lim _{x \rightarrow 0} f(x)=0$ in order to get to the point $S$ at the end of the limiting process, for small $x$ it must be of the form $f(x) \sim x^{\beta}$ with positive $\beta$. Let $f^{\prime}(x)$ denote the derivative of $f(x)$ with respect to its argument. Then, three situations can happen. We discuss them now.

\section{Slow approach to $S$ : Minkowski spacetime}

If $\lim _{x \rightarrow 0} x f^{\prime}(x) / f(x)<1$, then $y=f(x) \sim \alpha x^{1-\delta}$ (i.e., $\left.r_{0} / R \sim\left(q^{2} / R^{2}\right)^{1-\delta}\right)$, with positive $\alpha$ and $\delta$. Moreover, the mass goes as $m \sim x^{\delta} / 2 \alpha$ and vanishes in the limit $x \rightarrow 0$, so that the result in this case is the Minkowski spacetime. Finally, the Guilfoyle parameter $a$ is zero if $\delta>1 / 2$, is finite if $\delta=1 / 2$, and diverges if $\delta<1 / 2$.

\section{Linear approach to S: Schwarzschild black hole}

If $\lim _{x \rightarrow 0} x f^{\prime}(x) / f(x)=1$, then the function $y=f(x)$ tends to zero as $y=f(x) \sim \alpha x$ (i.e., $r_{0} / R \sim \alpha q^{2} / R^{2}$ ), with positive $\alpha$. In this case, $\lim _{x \rightarrow 0} m=\alpha^{-1} / 2$. Hence, $\lim _{x \rightarrow 0} r_{+}=2 m=\alpha^{-1}$ and $\lim _{x \rightarrow 0} r_{-}=0$. The metric functions are $B(r)=1 / A(r)=1-2 m / r$ for all $r>0$, the electric charge $q$ and the electric charge function $Q(r)$ vanish, and so does the electric potential $\phi(r)$. In such a case the result is a Schwarzschild black hole spacetime. The Guilfoyle parameter $a$ diverges.

Fast approach to $S:$ Kasner spacetime 
If $\lim _{x \rightarrow 0} x f^{\prime}(x) / f(x)>1$, then $y=f(x) \sim \alpha x^{1+\delta}$ (i.e., $\left.r_{0} / R \sim\left(q^{2} / R^{2}\right)^{1+\delta}\right)$, with positive $\alpha$ and $\delta$. The mass goes as $m \sim 1 /\left(2 \alpha x^{\delta}\right)$ and diverges in the limit $x \rightarrow 0$. This is exactly what happens when approaching the point $S$ along the line $r_{0}=0, q^{2}>0$, and the result in this case is a Kasner spacetime as shown in Sec. VM2 and in Appendix A2. The Guilfoyle parameter $a$ diverges.

\section{Synopsis}

As we have seen, for $q^{2} / R^{2}>0$, the mass function $m\left(q^{2}, r_{0}, R\right)$ may have different values at point $S$, the value it assumes there depends on how the point $S$ is approached. The limiting mass value may be zero, implying a Minkowski spacetime, it may be finite and nonzero, implying a Schwarzschild black hole, and it may diverge, implying a Kasner spacetime.

\section{Point $S$ from the left, $r_{0}=r_{+}=0, q^{2} \rightarrow 0^{-}:$Kasner spacetime}

When one approaches point $S$ from the left, i.e., performing $r_{0} / R=r_{-} / R=0$ and $q^{2} / R^{2} \rightarrow 0^{-}$, see Figs. 3 and 4 , one finds a Kasner spacetime. So the resulting spacetime is the same as found in Sec. VM2, by approaching point $S$ from the right performing $r_{0} / R=$ $r_{-} / R=0$ and $q^{2} / R^{2} \rightarrow 0^{+}$.

The limiting process is to take $r_{0} / R$ to zero, independently of $q^{2} / R^{2}$, with the constraint $q^{2} / R^{2}<0$, and then taking $q^{2} / R^{2}$ to zero. The first part of the process leads to a naked singularity metric with negative mass, and the second part of the process makes the charge and mass be transformed way by changing the coordinates. The result is then a naked planar singularity, more precisely, it is a static Kasner spacetime, see Sec. VL Appendix A1 and Appendix A2

5. Point $S$ from the skew left, $r_{0}>0, q^{2}<0$ : Minkowski spacetime, Schwarzschild negative mass singularity, and a plane-symmetric static spacetime naked singularity

\section{Generics}

When one approaches point $S$ from a skew path on the left, see Figs. 3 and 4, one finds, depending on the approaching path, a Minkowski spacetime, or a Schwarzschild black hole, or a Kasner spacetime. All the three cases have zero electric charge and zero electric potential, of course, since point $S$ has $q^{2}=0$.

The analysis of Sec. VM3 is also appropriate here, since the behavior of the mass function when reaching the point $S$ from negative values of $q^{2}<0$ is similar as for the $q^{2}>0$ case. We define here $x \equiv|q|^{2} / R^{2}$ and $y \equiv r_{0} / R$. Let $\gamma$ be a a path in the two-dimensional parameter space spanned by $x$ and $y$. Along $\gamma$ we may write $y=f(x)$ with $\gamma$ being parametrized by $x$. Assume $f$ to be a piecewise continuous function of its argument. Since $f(x)$ has also to satisfy the condition $\lim _{x \rightarrow 0} f(x)=0$ in order to get to the point $S$ at the end of the limiting process, for small $x$ it must be of the form $f(x) \sim x^{\beta}$ with positive $\beta$. Let $f^{\prime}(x)$ denote the derivative of $f(x)$ with respect to its argument. Then again, three situations can happen. We discuss them now.

\section{Slow approach to $S$ : Minkowski spacetime}

If $\lim _{x \rightarrow 0} x f^{\prime}(x) / f(x)<1$, then $f(x) \sim x^{1-\delta}$ (i.e., $r_{0} / R \sim$ $\left.\left(|q|^{2} / R^{2}\right)^{1-\delta}\right)$, with positive $\alpha$ and $\delta$, the mass goes as $m \sim-x^{\delta} / 2 \alpha$, so is negative, and vanishes in the limit $x \rightarrow 0$. The result in this case is the Minkowski spacetime.

Linear approach to S: Schwarzschild negative mass singularity

If $\lim _{x \rightarrow 0} x f^{\prime}(x) / f(x)=1$, then the function $f(x)$ tends to zero as $y=f(x) \sim \alpha x$ (i.e., $r_{0} / R \sim \alpha q^{2} / R^{2}$ ), with positive $\alpha$, and we have $\lim _{x \rightarrow 0} m=-\alpha^{-1} / 2$, so the mass is negative. There are no horizons. The metric functions result in $B(r)=1 / A(r)=1+2|m| / r$ for all $r>0$, the electric charge $q$ and the electric charge function $Q(r)$ vanish, and so does the electric potential $\phi(r)$. In such a case there are no horizons and the result is a Schwarzschild negative mass singularity.

\section{Fast approach to $S:$ A plane-symmetric static spacetime naked singularity}

If $\lim _{x \rightarrow 0} x f^{\prime}(x) / f(x)>1$, then the function $f(x)$ tends to zero as $y=f(x) \sim \alpha x^{1+\delta}$ (i.e., $r_{0} / R \sim \alpha\left(|q|^{2} / R^{2}\right)^{1+\delta}$ ), with positive $\alpha$ and $\delta$, and the mass goes as $m \sim$ $-1 /\left(2 \alpha x^{\delta}\right)$ which is negative and diverges in the limit $x \rightarrow 0$. This is exactly what happens along the line $r_{0}=$ $0, q^{2}>0$, but now the mass is negative, so the metric assumes the form $d s^{2}=-2|m| d t^{2} / r+r d r^{2} /(2|m|)+r^{2} d \Omega^{2}$. One can then transform this metric away into a more common plane symmetric static naked singularity metric form, more precisely, it is a static Kasner spacetime, see $\mathrm{Sec} \mathrm{VH}$ and Appendix A1.

\section{Synopsis}

As we have seen, for $q^{2} / R^{2}<0$, the mass function $m\left(q^{2}, r_{0}, R\right)$ assumes at the point $S$ different values depending on how the point $S$ is approached. In terms of the resulting spacetime, the limit is three-folded. The limiting mass value may be zero, implying the Minkowski 
spacetime, may be finite and nonzero, implying a negative mass naked Schwarzschild singularity, and may diverge, resulting in a planar static naked singularity Kasner type spacetime.

\section{N. Point B: The Buchdahl bound}

Point $B$ is the Buchdahl bound [32], here represented by a Schwarzschild star (i.e., a Schwarzschild interior solution matched to the Schwarzschild vacuum solution) for which $r_{0} / m=9 / 4$, see Figs. 3 and 4 .

Point $B$ is the zero electric charge, i.e., $q^{2}=0$, limit of the Buchdahl-Andréasson bound curve $C_{4}$, see Sec. IIID 2, particularly Eq. (67), and Sec. (VE) (for a detailed study of the Buchdahl-Andréasson bound see [4]). The features of point $B$ may be found by taking the limit $q^{2} / R^{2} \rightarrow 0$ and then by putting the central pressure to infinity in the solutions. This furnishes $r_{0} / R=2 \sqrt{2} / 3$. The limit for the mass function gives $m\left(0, r_{0}, R\right)=r_{0}^{3} / 2 R^{2}$ and then we get the Buchdahl limit in the usual form, $r_{0} / m=9 / 4$.

\section{O. Point Q, $q=m=r_{+}=r_{-}=r_{0}=R$ : Quasiblack holes}

Point $Q$ represents quasiblack hole configurations, i.e., extremal charged stars that are on the verge of being a black hole [3], see Figs. 3 and 4 .

Point $Q$ is found by taking the limits $q / R \rightarrow 1$ and $r_{0} / R \rightarrow 1$, and it gives $q=m=r_{+}=r_{-}=r_{0}=R$. Point $Q$ belongs to the boundary of four very different regions: Region (a) with its undercharged regular stars, region (b) of the overcharged regular stars, region (c) of overcharged singular solutions, regions (d) and (e) of regular black holes. It is also the convergence of several curves or lines: The Buchdahl-Andréasson curve $C_{4}$, the dust curve $C_{0}$, the curve $C_{1}$ of null Guilfoyle parameter $a=0$, the second branch of the dust curve $C_{2}$, the curve $C_{3}$, the line $r_{+}=r_{0}=R$, and the line of regular black holes with matching at the inner horizon $r_{0}=r_{-}=$ $R$. This means that the solutions corresponding to this point are very special and highly degenerated, they are quasiblack holes, see also Sec. [IID 5] and [3].

\section{CONCLUSIONS}

We have explored the full parameter space of Guilfoyle's exact solutions for relativistic charged spheres [1].
There are three free parameters among the various parameters of the model. We have chosen the normalized charge squared parameter $q^{2} / R^{2}$ and the normalized radius of the spheres $r_{0} / R$ as the good normalized parameters, where $R$ is the third free parameter characterizing the constant energy-density of the model, $8 \pi \rho_{m}(r)+Q^{2}(r) / r^{4}=3 / R^{2}$. In order to avoid spacelike matching, the ratio $r_{0} / R$ is constrained in the interval $0 \leq r_{0} / R \leq 1$. The limiting value $r_{0} / R=1$ implies the matching surface is lightlike, while $r_{0} / R<1$ leads to different kinds of spacetime. We allow the parameter $q^{2} / R^{2}$ to assume all values in the real line. When $q^{2}$ is negative the interpretation of the parameter $q$ as electric charge is not possible. However, $q^{2}$ may be interpreted as a tidal charge in a braneworld gravity. The other two parameters, the mass $m$ and the Guilfoyle parameter $a$ depend on the three free parameters mentioned above. The Guilfoyle parameter $a$ relates the metric to the gauge potentials. Allowing $a$ to run along all values of the real line has proved essential in the search for new solutions.

The plethora of solutions found has been proved to bear very interesting spacetimes. Besides the wellbehaved electrically charged stars examined in the original work [1], for which it was shown that the fluid content has precise algebraic properties [2], there are quasiblack holes with pressure [3], there are charged stars that saturate the Buchdahl-Andréasson bound [4] when the central pressure is allowed to reach arbitrarily large values, in the very same way the Schwarzschild interior solutions saturate the Buchdahl bound in the limit that the central pressure diverges, there are regular black holes with a de Sitter core and a coat of electric charge, regular black holes with a core of charged phantom fluid [5], other exotic regular black holes, as well as other solutions. This shows that the full spectrum presents a bewildering variety of possible solutions, from all types of stars to all types of black holes, though wormholes do not appear in the spectrum. A stability analysis of Guilfoyle's solutions is yet to be performed.

\section{Appendix A: Planar naked negative mass singularity and Kasner metrics: Details}

\section{1. $r_{0}=r_{+}=0, q^{2}<0$ : Planar naked negative mass singularities}

In the $r_{0}=r_{+}=0$ case, discussed in Sec. IIID3 for a fixed value of $q^{2} / R^{2}<0$ one finds that the spacetime for small $r$ has planar symmetry and is singular at $r=0$. To see this, let us analyze the limit $r_{0} / R \rightarrow 0$ in the Guilfoyle solutions for a fixed value of $q^{2} / R^{2}<0$.

Putting $r_{0} / R \rightarrow 0$ in the mass function, Eq. (34), one 
finds

$$
m=-\frac{|q|^{2}}{2 r_{0}}+\mathrm{O}\left(\frac{r_{0}^{2}}{|q|^{2}}\right)^{3 / 2} .
$$

So, the mass is negative and divergent in this limit.

Putting $r_{0} / R \rightarrow 0$ in the equation for the Guilfoyle parameter $a$, Eq. (35), one finds

$$
a=-\frac{|q|^{2}}{4 r_{0}^{2}}-\frac{|q|^{2}}{4}+\mathrm{O}\left(\frac{r_{0}}{R}\right)^{2} .
$$

So, $a$ is negative and divergent in this limit.

Putting $r_{0} / R \rightarrow 0$ in the equation for the gravitational radius $r_{+}$, Eq. (32), using Eq. (34), and $q^{2}=-|q|^{2}$, yields

$$
r_{+}=r_{0}-\mathrm{O}\left(\frac{r_{0}^{2}}{|q|^{2}}\right)^{7 / 2}
$$

Hence, in this limit the gravitational radius $r_{+}$goes to zero as $r_{0}$, giving rise to a spacetime singularity at $r=$ $r_{0}=r_{+}=0$.

Putting $r_{0} / R \rightarrow 0$ in the equation for the Cauchy radius $r_{-}$, Eq. (33), using Eq. (34), and $q^{2}=-|q|^{2}$, yields

$$
r_{-}=-\frac{|q|^{2}}{2 r_{0}}+r_{0}+\mathrm{O}\left(\frac{r_{0}^{2}}{|q|^{2}}\right)^{3 / 2} .
$$

Hence, in this limit the Cauchy radius is negative and of no interest at all, since we are considering solutions with $r \geq 0$.

To interpret the resulting metric note that for $r_{0}$ very small but not zero, the metric for $r>r_{0}$ is the ReissnerNordström metric with arbitrarily large and negative $m$, from Eq. A1). This is equivalent to take the limit of large $-m$ with finite $q^{2}<0$ in the Reissner-Nordström metric, Eqs. (11), (24) and (25), yielding

$$
d s^{2}=-\frac{2|m|}{r} d t^{2}+\frac{r d r^{2}}{2|m|}+r^{2}\left(d \theta^{2}+\sin ^{2} \theta d \varphi^{2}\right) .
$$

This metric is the negative mass Schwarzschild black hole metric close to the singularity. It can be put into a form that makes explicit the planar symmetry. Defining a new spacelike coordinate $l$ such that $d l^{2}=r d r^{2} / 2|m|$ we get $d s^{2}=-\left(l / l_{0}\right)^{-2 / 3} d t^{2}+d l^{2}+\left(l / l_{0}\right)^{4 / 3}\left(4|m|^{2}\right)\left(d \theta^{2}+\right.$ $\left.\sin ^{2} \theta d \varphi^{2}\right)$, where we defined $l_{0}=4|m| / 3$. To complete the transformation into a plane-symmetric metric, note that at a given point with coordinates $\left(t, l, \theta_{0}, \varphi_{0}\right)$ we may define local Cartesian coordinates $x=2 m\left(\theta-\theta_{0}\right)$ and $y=2 m\left(\varphi-\varphi_{0}\right) \sin \theta_{0}$, where $(\theta, \varphi)$ are in the neighborhood of $\left(\theta_{0}, \varphi_{0}\right)$, so the metric results in

$$
d s^{2}=-\left(\frac{l}{l_{0}}\right)^{-2 / 3} d t^{2}+d l^{2}+\left(\frac{l}{l_{0}}\right)^{4 / 3}\left(d x^{2}+d y^{2}\right)
$$

which shows the planar symmetry of the surfaces defined by $t, l=$ constant. This metric can be considered a static Kasner metric (see, e.g., [45, 46]), derived from the usual Kasner metric by a complex transformation that swaps the time coordinate with the radial one [46], with characteristic Kasner parameters given by $p_{1}=-1 / 3$, and $p_{2}=p_{3}=2 / 3$. The radius of the matter distribution region $r_{0}$ can be finally set to zero, so that the two-dimensional timelike boundary surface $r=r_{0}=r_{+}$ flattens to a plane at $l=0$, giving rise to a timelike planesymmetric singularity. The plane symmetric singularity at $l=0$ is naked.

\section{2. $r_{0}=r_{-}=\mathbf{0}, q^{2}>0$ : Kasner spacetimes}

In the $r_{0}=r_{-}=0$ case, discussed in Sec. IIID 4, for a fixed value of $q^{2} / R^{2}<0$ one finds that the spacetime for small $r$ is a Kasner spacetime. To see this, let us analyze the limit $r_{0} / R \rightarrow 0$ in the Guilfoyle solutions for a fixed value of $q^{2} / R^{2}<0$.

Putting $r_{0} / R \rightarrow 0$ in the mass function, Eq. (34), one finds

$$
m=\frac{q^{2}}{2 r_{0}}+\mathrm{O}\left(\frac{r_{0}^{2}}{q^{2}}\right)^{3 / 2} .
$$

So, for finite charge and in the limit $r_{0} / R \rightarrow 0$, the mass inside $r_{0}$ is positive and diverges giving rise to a spacetime singularity at $r_{-}=r_{0}=0$.

Putting $r_{0} / R \rightarrow 0$ in the equation for the Guilfoyle parameter $a$, Eq. (35), one finds

$$
a=\frac{q^{2}}{4 r_{0}^{2}}+\frac{q^{2}}{4}+\mathrm{O}\left(\frac{r_{0}}{R}\right)^{2} .
$$

So, $a$ is positive and divergent in this limit.

Putting $r_{0} / R \rightarrow 0$ in the equation for the gravitational radius $r_{+}$, Eq. (32), using Eq. (34) for $q^{2}>0$, yields

$$
r_{+}=\frac{q^{2}}{2 r_{0}}-r_{0}+\mathrm{O}\left(\frac{r_{0}^{2}}{q^{2}}\right)^{3 / 2} .
$$

Hence, in this limit the gravitational radius $r_{+}$diverges.

Putting $r_{0} / R \rightarrow 0$ in the equation for the Cauchy radius $r_{-}$, Eq. (33), using Eq. (34) for $q^{2}$, yields

$$
r_{-}=r_{0}+\mathrm{O}\left(\frac{r_{0}^{2}}{q^{2}}\right)^{7 / 2} .
$$

Hence, in this limit the Cauchy radius goes to zero as $r_{0}$, it disappears.

To interpret the resulting metric note that for $r_{0}$ very small but not zero, the metric for $r>r_{0}$ is the ReissnerNordström metric with arbitrarily large and positive $m$, 
from Eq. (A1). This is equivalent to take the limit of large $m$ with finite $q^{2}>0$ in the Reissner-Nordström metric, Eqs. (1), (24) and (25), yielding

$$
d s^{2}=+\frac{2 m}{r} d t^{2}-\frac{r d r^{2}}{2 m}+r^{2}\left(d \theta^{2}+\sin ^{2} \theta d \varphi^{2}\right) .
$$

This metric is the Schwarzschild black hole metric close to the singularity. It can be put into a form that is locally a Kasner metric (see, e.g., [45]). Defining a new timelike coordinate $\tau$ such that $d \tau^{2}=r d r^{2} / 2 m$, and a new spacelike coordinate $r$ by $r=t$ we get $d s^{2}=$ $-d \tau^{2}+\left(\tau / \tau_{0}\right)^{-2 / 3} d r^{2}+\left(\tau / \tau_{0}\right)^{4 / 3}\left(4 m^{2}\right)\left(d \theta^{2}+\sin ^{2} \theta d \varphi^{2}\right)$, where we defined $\tau_{0}=4 \mathrm{~m} / 3$. To complete the transformation into a plane-symmetric metric, note that at a given point with coordinates $\left(t, l, \theta_{0}, \varphi_{0}\right)$ we may define local Cartesian coordinates $x=2 m\left(\theta-\theta_{0}\right)$ and $y=2 m\left(\varphi-\varphi_{0}\right) \sin \theta_{0}$, where $(\theta, \varphi)$ are in the neighborhood of $\left(\theta_{0}, \varphi_{0}\right)$, so the metric results in

$$
d s^{2}=-d \tau^{2}+\left(\frac{\tau}{\tau_{0}}\right)^{-2 / 3} d r^{2}+\left(\frac{\tau}{\tau_{0}}\right)^{4 / 3}\left(d x^{2}+d y^{2}\right)
$$

which is the Kasner universe metric with characteristic Kasner parameters given by $p_{1}=-1 / 3$, and $p_{2}=p_{3}=$ $2 / 3$ (see, e.g., [45, 46]). The radius of the matter distribution $r_{0}$ can be finally set to zero, so that the two- dimensional timelike boundary surface at $r=r_{0}$ flattens to a plane containing an infinite mass, giving rise to a spacelike singularity. The singularity is at $\tau=0$, a bigbang or a big-crunch like singularity. The surfaces $\tau$, $r=$ constant are now two-dimensional spacelike planes.

\section{Acknowledgments}

We thank Fundação para a Ciência e Tecnologia (FCT), Portugal, for financial support through Grant No. UID/FIS/00099/2013. This work was partially funded by Coordenação de Aperfeiçoamento do Pessoal de Nível Superior (CAPES), Brazil, Grants No. 88881.064999/2014-01 and Grant No. 88887.068694/2014-00. J. P. S. L. also thanks an FCT grant, No. SFRH/BSAB/128455/2017, and Piotr Chruściel and the Gravitational Physics Group at the Faculty of Physics, University of Vienna, for hospitality. V. T. Z. thanks Fundação de Amparo à Pesquisa do Estado de São Paulo (FAPESP), Grant No. 2011/18729-1, and Conselho Nacional de Desenvolvimento Científico e Tecnológico of Brazil (CNPq), Grant No. 308346/2015-7.
[1] B. S. Guilfoyle, "Interior Weyl-type solutions of the Einstein-Maxwell field equations", Gen. Relativ. Gravit. 31, 1645 (1999); arXiv:gr-qc/9906089.

[2] J. P. S. Lemos and V. T. Zanchin, "Electrically charged fluids with pressure in Newtonian gravitation and general relativity in $\mathrm{d}$ spacetime dimensions: Theorems and results for Weyl type systems", Phys. Rev. D 80, 024010 (2009); arXiv:0905.3553 [gr-qc].

[3] J. P. S. Lemos and V. T. Zanchin, "Quasiblack holes with pressure: Relativistic charged spheres as the frozen stars", Phys. Rev. D 81, 124016 (2010); arXiv:1004.3574 [gr-qc].

[4] J. P. S. Lemos and V. T. Zanchin, "Sharp bounds on the radius of relativistic charged spheres: Guilfoyle's stars saturate the Buchdahl-Andréasson bound", Classical Quantum Gravity 32, 135009 (2015); arXiv:1505.03863 [gr-qc].

[5] J. P. S. Lemos and V. T. Zanchin, "Regular black holes: Guilfoyle electrically charged solutions with a perfect fluid phantom core", Phys. Rev. D 93, 124012 (2016); arXiv:1603.07359 [gr-qc].

[6] H. Weyl, Zur Gravitationstheorie, Ann. Phys. (Berlin) 359, 117 (1917).

[7] S. D. Majumdar, "A class of exact solutions of Einstein's field equations", Phys. Rev. 72, 390 (1947).

[8] A. Papapetrou, "A static solution of the equations of the gravitational field for an arbitrary charge-distribution", Proc. R. Irish Acad. A 51, 191 (1947).

[9] J. P. S. Lemos and V. T. Zanchin, "A class of exact solutions of Einstein's field equations in higher dimensional spacetimes, d $\geq 4$ : Majumdar-Papapetrou solutions", Phys. Rev. D 71, 124021 (2005); arXiv:gr-qc/0505142.

[10] W. B. Bonnor and S. B. P. Wickramasuriya, "Are very large gravitational redshifts possible", Mon. Not. R. Astron. Soc. 170, 643 (1975).

[11] J. P. S. Lemos and V. T. Zanchin, "Bonnor stars in $d$ spacetime dimensions", Phys. Rev. D 77, 064003 (2008); arXiv:0802.0530 [gr-qc].

[12] F. I. Cooperstock and V. de la Cruz, "Sources for the Reissner-Nordström metric", Gen. Relativ. Gravit. 9, 835 (1978).

[13] P. S. Florides, "The complete field of charged perfect fluid spheres and of other static spherically symmetric charged distributions", J. Phys. A 16, 1419 (1983).

[14] A. Das, "A class of exact solutions of certain classical field equations in general relativity", Proc. R. Soc. A 267, 1 (1962).

[15] U. K. De and A. K. Raychaudhuri, "Static distribution of charged dust in general relativity", Proc. R. Soc. A 303, 97 (1968).

[16] R. Gautreau and R. B. Hoffman, "The structure of the sources of Weyl-type electrovac fields in general relativity", Nuovo Cimento B 16, 162 (1973).

[17] W. B. Bonnor, "Equilibrium of charged dust in general relativity", Gen. Relativ. Gravit. 12, 453 (1980).

[18] J. P. S. Lemos and E. J. Weinberg, "Quasiblack holes from extremal charged dust", Phys. Rev. D 69, 104004 (2004); arXiv:gr-qc/0311051.

[19] A. Kleber, J. P. S. Lemos, and V. T. Zanchin, "Thick shells and stars in Majumdar-Papapetrou general rel- 
ativity", Gravit. Cosmol. 11, 269 (2005); arXiv:grqc/0406053.

[20] J. P. S. Lemos and V. T. Zanchin, "Gravitational magnetic monopoles and Majumdar-Papapetrou stars", J. Math. Phys. 47, 042504 (2006); arXiv:gr-qc/0603101.

[21] J. P. S. Lemos and O. B. Zaslavskii, "Quasiblack holes: Definition and general properties", Phys. Rev. D 76, 084030 (2007); arXiv:0707.1094 [gr-qc].

[22] J. P. S. Lemos and O. B. Zaslavskii, "Black hole mimickers: Regular versus singular behavior", Phys. Rev. D 78, 024040 (2008); arXiv:0806.0845 [gr-qc].

[23] J. P. S. Lemos and O. B. Zaslavskii, "The mass formula for quasiblack holes", Phys. Rev. D 78, 124013 (2008); arXiv:0811.2778 [gr-qc].

[24] J. P. S. Lemos and O. B. Zaslavskii, "Entropy of quasiblack holes", Phys. Rev. D 81, 064012 (2010); arXiv:0811.2778 [gr-qc].

[25] J. P. S. Lemos and O. B. Zaslavskii, "Quasiblack holes with pressure: General results", Phys. Rev. D 82, 024029 (2010); arXiv:1004.4651 [gr-qc].

[26] W. B. Bonnor and S. B. P. Wickramasuriya, "A static body of arbitrarily large density", Int. J. Theor. Phys. 5, 371 (1972).

[27] F. de Felice, Y. Yunqiang, and F. Jing, "Relativistic charged spheres", Mon. Not. R. Astron. Soc. 277, L17 (1995).

[28] F. de Felice, L. Siming, and Y. Yunqiang, "Relativistic charged spheres: II. Regularity and stability", Classical Quantum Gravity 16, 2669 (1999); arXiv:gr-qc/9905099.

[29] W. B. Bonnor, "Comment on 'Relativistic charged spheres: II. Regularity and stability' ", Classical Quantum Gravity 16, 4125 (1999).

[30] A. Lue and E. J. Weinberg, "Magnetic monopoles near the black hole threshold", Phys. Rev. D 60, 084025 (1999); arXiv:hep-th/9905223.

[31] R. Meinel, "On the black hole limit of rotating fluid bodies in equilibrium ", Classical Quantum Gravity 23, 1359 (2006); arXiv:gr-qc/0506130.

[32] H. A. Buchdahl, "General relativistic fluid spheres", Phys. Rev. 116, 1027 (1959).

[33] H. Andréasson, "Sharp bounds on the critical stability radius for relativistic charged spheres", Commun. Math. Phys. 288, 715 (2009); arXiv:0804.1882 [gr-qc].

[34] J. D. V. Arbañil, J. P. S. Lemos, and V. T. Zanchin, "Incompressible relativistic spheres: Electri- cally charged stars, compactness bounds, and quasiblack hole configurations". Phys. Rev. D 89, 104054 (2014); arXiv:1404.7177 [gr-qc].

[35] J. P. S. Lemos, F. J. Lopes, G. M. Quinta, and V. T. Zanchin, "Compact stars with a small electric charge: the limiting radius to mass relation and the maximum mass for incompressible matter". Europ. Phys. J. C 12, 3456 (2015); arXiv:1408.1400 [astro-ph.SR].

[36] J. M. Bardeen, "Non-singular general-relativistic gravitational collapse", in Abstracts of the 5th International Conference on Gravitation and the Theory of Relativity, edited by V. A. Fock (Tbilisi University Press, Tbilisi, 1968), p. 174.

[37] I. G. Dymnikova, "Vacuum nonsingular black hole", Gen. Relativ. Gravit. 24, 235 (1992).

[38] E. Ayón-Beato and A. García, "The Bardeen model as a nonlinear magnetic monopole", Phys. Lett. B 493, 149 (2000); arXiv:gr-qc/0009077.

[39] K. A. Bronnikov and J. C. Fabris, "Regular phantom black holes", Phys. Rev. Lett. 96, 251101 (2006); arXiv:gr-qc/0511109.

[40] J. Matyjasek, D. Tryniecki, and M. Klimek, "Regular black holes in an asymptotically de Sitter universe", Mod. Phys. Lett. A 23, 3377 (2008); arXiv:0809.2275 [gr-qc].

[41] J. P. S. Lemos and V. T. Zanchin, "Regular black holes: Electrically charged solutions, Reissner-Nordström outside a de Sitter core", Phys. Rev. D 83, 124005 (2011); arXiv:1104.4790 [gr-qc].

[42] A. B. Balakin, J. P. S. Lemos, and A. E. Zayats, "Regular nonminimal magnetic black holes in spacetimes with a cosmological constant", Phys. Rev. D 93, 024008 (2016); arXiv:1512.02653 [gr-qc].

[43] A. Einstein, N. Rosen, "The particle problem in the general theory of relativity", Phys. Rev. 48, 73 (1935).

[44] N. Dadhich, R. Maartens, P. Papadopoulos, and V. Rezania, "Black holes on the brane", Phys. Lett. B 487, 1 (2000); arXiv:hep-th/0003061.

[45] W. A. Hiscock, S. L. Larson, and P. R. Anderson, "Semiclassical effects in black hole interiors", Phys. Rev. D 56, 3571 (1997), arXiv:gr-qc/9701004.

[46] J. B. Griffiths and J. Podolský, Exact space-times in Einstein's general relativity (Cambridge University Press, Cambridge, 2009). 